\title{
A mechanism for hot spot generation in a reactive two-dimensional sheared viscous layer
}

\author{
Robert Timms ${ }^{a *}$, Richard Purvis $^{a}$ and John P. Curtis ${ }^{b, c}$ \\ ${ }^{a}$ School of Mathematics, University of East Anglia, Norwich NR4 7TJ, UK; \\ ${ }^{b} A W E$, Aldermaston, Reading, Berkshire RG7 $4 P R, U K$; \\ ${ }^{c}$ Department of Mathematics, University College London, Gower Street, London WC1E \\ $6 B T, U K$
}

\begin{abstract}
A two-dimensional model for the non-uniform melting of a thin sheared viscous layer is developed. An asymptotic solution is presented for both a non-reactive and a reactive material. It is shown that the melt front is linearly stable to small perturbations in the non-reactive case, but becomes linearly unstable upon introduction of an Arrhenius source term to model the chemical reaction. Results demonstrate that non-uniform melting acts as a mechanism to generate hot spots which are found to be sufficient to reduce the time to ignition when compared with the corresponding one-dimensional model of melting.
\end{abstract}

Keywords: Hot spot; Ignition; Shear-melting;

\section{Introduction}

Safety is of paramount importance in the handling, processing and storage of explosives. While much is known about the behaviour of explosive materials during high speed deformation, the mechanisms responsible for the ignition of explosive materials in response to low energy stimuli, known as 'insults' in the literature, are still not well understood. It is in general believed that explosive ignition is of thermal origin, with mechanical energy being converted into heat energy in localised regions, forming 'hot spots' [1]. Many hot spot mechanisms have been proposed in the literature, but it is found that the explosive response is strongly dependent on the material properties and type of insult, making it difficult to identify a single mechanism as dominant. The main mechanisms suggested for hot spot generation include adiabatic compression of trapped gas spaces, viscous heating of rapidly extruded material, friction between impacting surfaces, local adiabatic shear, and heating at crack tips. Note that this list is certainly not exhaustive and many more mechanisms have been described in other studies [see 1, and references therein].

In particular, mechanisms arising from shear are widely discussed in the literature. For instance, $[2-5]$ discuss frictional rubbing as an ignition mechanism. During rubbing contact between two solids, the hot spot temperature is determined by the solid with the lower melting point - the lower melting point solid 'quenches' the hot spot temperature to the melting temperature. Bowden and Gurton were able to

*Corresponding author. Email: r.timms@uea.ac.uk 
measure hot spot ignition temperatures for a wide range of explosives by choosing grits of different melting points and measuring the effect on the sensitivities of the explosives [4].

While shear localisation has been widely studied in inert materials (see $[6,7]$, for example), there have been very few analytical studies on localised shear in explosive materials. In contrast to the dearth of analytical work, many experimental studies can be found in the literature. Evidence for localised shear within the explosive sample can be observed in recovered unexploded samples. Photographic evidence for adiabatic shear is given by Field et al. [8], showing ignition and propagation occurring in a shear band in a sample of PETN (pentaerythritol tetranitrate). Notable analytical work on shear localisation in explosive materials includes [912 ] and the substantial work [13]. Also worthy of mention are the experimental works $[14,15]$. It is in general concluded that localised shear is a prevalent hot spot mechanism, which manifests in many differing loading scenarios.

Investigation of explosive response to insults through the use of numerical continuum mechanics methods, such as finite element models, often falls victim to problems such as severe mesh deformation [16]. Typically, a very high resolution is required to overcome such issues, but this comes at the cost of computational resources and time. Additionally, large scale numerical codes do not always offer as much physical insight as small scale, simplified, analytical models. Here we develop a simplified model for the shearing and subsequent melting of a solid block of explosive material to try and gain a deeper understanding of the mechanisms which may lead to thermal runaway.

We consider the melting of a thin viscous layer of explosive material due to an applied shear. When an explosive sample is subject to a mechanical insult preexisting, or new, microcracks will be in compression and shear. Such microcracks can grow in size if the local stress is great enough and, due to friction between solid surfaces, heat is released during the growth process [17]. Subsequent to sufficient heat release, the crack surface temperature will be raised to the solid melting point and a thin sheared melt layer will be formed, separating the solid surfaces [18]. This thin melt layer will continue to be heated through viscous dissipation and is thought to be a prime location for hot spot generation.

Starobin and Dienes [17] develop a one-dimensional model for the lateral melting and ignition of a thin, sheared, viscous layer of the reactive solid HMX (cyclotetramethylene tetranitramine). In their work, they present an analytical and numerical study of the melt front propagation both with and without a chemical reaction. Assuming a melt layer of uniform width, and adopting a non-linear Arrhenius heat source term to capture the reaction, they demonstrate that shear melting in the one-dimensional geometry leads to an increase in the peak temperature relative to the melting point of HMX.

A natural question arises: will the typically inhomogeneous structure found in explosive materials cause further localisation? Polymer bonded explosives are highly granular materials, and the constituent materials in the explosive will in general have different thermomechanical properties. Such spatial differences in material properties will inevitably create a non-uniform melt layer. In this paper, we formally derive a two-dimensional model for the melting and ignition of a thin layer of explosive material, so that the effects of material inhomogeneity can be investigated. In order to account for the possible non-uniform melting of material, we impose a shape on the melt front which is allowed to evolve in time. In particular, we demonstrate that even a small two-dimensional disturbance can lead to the generation of highly localised hot spots within the melt layer and greatly reduce the predicted time to runaway. 
In [17] all material properties are treated as constant, allowing one reasonably to assume a linear velocity profile across the melt layer to leading-order. However, the temperature across the layer varies from the melt temperature of $520 \mathrm{~K}$ to a peak temperature of well over $700 \mathrm{~K}$. Inevitably this large increase in temperature will have some effect on the material properties. In particular, it is suggested that the viscosity may vary significantly across the layer, and the linearisation of the fluid velocity across the layer would break down. Here we allow the specific heat, viscosity and thermal conductivity to vary with temperature and compare the results with those found in [17].

In Section 2 we derive a two-dimensional model describing the heating of thin molten layer of explosive undergoing shear. The thinness of the melt layer is exploited and a lubrication approximation is made. The resulting equations are transformed into front-fixed coordinates, which are particularly convenient for the numerical analysis to follow. In Section 3 we investigate the effects arising from the two-dimensionality of our model, and make the simplifying assumption of constant material properties. A linear stability analysis in conducted, considering small deviations from a melt layer of uniform width. It is demonstrated the the melt front is linearly stable in the absence of any chemical reaction, but stability is lost upon introduction of an Arrhenius heat source which models the chemical reactions occurring within the layer. In Section 3.2 we present an iterative numerical scheme which is used to determine the temperature field in the melt layer. Times to ignition are computed for various initial conditions, demonstrating the effect the imposed melt front shape has on the self heating in the layer. In Section 4 we investigate the effects of allowing the liquid specific heat, viscosity and thermal conductivity to vary with temperature. We demonstrate a departure from the leading-order linear velocity profile found in [17], and show this leads to non-uniform mechanical heating across the layer. We conclude in Section 5, where we discuss the possible physical interpretations of the model and offer some suggestions for the conditions under which non-uniform melting may occur.

\section{Model equations}

We consider a semi-infinite solid block of explosive material occupying the the region $x>0$, with a rigid wall located at $x=0$. At time $t=0$ the wall moves impulsively downwards with speed $v_{\mathrm{w}}$. The movement provides a shear force on the explosive sample, generating sufficient heat to melt the explosive sample near the wall, such that, at $t=t_{0}$, there already exists a thin viscous liquid melt layer adjacent to the wall, see Figure 1. The melt front, shown here as a dashed line, is located at $x=x_{f}(y, t)$.

A possible physical interpretation of the current work is to consider a scenario in which an internal crack develops, and two material planes slide against one another. In this case, the model is readily adapted to account for a symmetry condition at $x=0$, as in [17]. The solid wall used in the current formulation may also be considered as a model for a piece of high melting point grit present in the explosive sample [3]. In any case, the heating mechanisms discussed here are still present.

The molten explosive in the melt layer is modelled as an incompressible viscous fluid with temperature dependent specific heat $c$, viscosity $\mu$ and thermal conductivity $\kappa$. Under these assumptions, the continuity equation and Navier-Stokes 
equations, given here in non-dimensional form, are

$$
\begin{aligned}
\frac{\partial u}{\partial x}+\frac{\partial v}{\partial y} & =0 \\
\operatorname{Re}\left(\frac{\partial u}{\partial t}+u \frac{\partial u}{\partial x}+v \frac{\partial u}{\partial y}\right) & =-\frac{\partial p}{\partial x}+2 \frac{\partial}{\partial x}\left(\mu \frac{\partial u}{\partial x}\right)+\frac{\partial}{\partial y}\left[\mu\left(\frac{\partial u}{\partial y}+\frac{\partial v}{\partial x}\right)\right], \\
\operatorname{Re}\left(\frac{\partial v}{\partial t}+u \frac{\partial v}{\partial x}+v \frac{\partial v}{\partial y}\right) & =-\frac{\partial p}{\partial y}+2 \frac{\partial}{\partial y}\left(\mu \frac{\partial v}{\partial y}\right)+\frac{\partial}{\partial x}\left[\mu\left(\frac{\partial u}{\partial y}+\frac{\partial v}{\partial x}\right)\right],
\end{aligned}
$$

where $(u, v)$ are the horizontal and vertical components of the liquid velocity, respectively, $p$ is the pressure and $\operatorname{Re}=\left(\rho v^{*} l^{*}\right) / \mu^{*}$ is the Reynolds number. Here we have non-dimensionalised using typical velocity scale $v^{*}=v_{\mathrm{w}}$, time scale $t^{*}$, density $\rho$ and reference viscosity $\mu^{*}$. The length scale may be given as the product of a time scale $t^{*}$ and the typical wall speed $v_{\mathrm{w}}$, i.e. $l^{*}=v_{\mathrm{w}} t^{*}$. The pressure has been scaled with $\left(\mu^{*} v_{\mathrm{w}}\right) / l^{*}$ to balance with the viscous terms. As in [17], we assume the molten explosive behaves as a viscous fluid, so appropriate conditions on the wall and liquid/solid boundary are the no-slip conditions:

$$
\begin{array}{lll}
u=0, & v=-1 & \text { on } x=0, \\
u=0, & v=0 & \text { on } x=x_{f}(y, t) .
\end{array}
$$

The explosive sample is initially heated by viscous dissipation in the melt layer and, once the temperature rise is sufficient, heated further by a consequent chemical reaction. We denote the temperature in the melt layer by $T$ and the solid temperature by $T_{\text {sol }}$. The chemical reaction is modelled as a single step Arrhenius reaction, expressed in terms of the mass fraction $\alpha$ of gaseous products [16]. This is given in non-dimensional form by

$$
\frac{\partial \alpha}{\partial t}+u \frac{\partial \alpha}{\partial x}+v \frac{\partial \alpha}{\partial y}=\hat{A}(1-\alpha) \exp \left(-\frac{\hat{E}}{T}\right)
$$

where $\hat{A}=t^{*} A=\left(l^{*} A\right) / v_{\mathrm{w}}$ is the non-dimensional pre-exponential factor, $\hat{E}=$ $E /(R \Delta T)$ is the non-dimensional activation energy, $E$ is the activation energy, $R$ is the molar gas constant and $\Delta T$ a typical temperature difference. It is well known that multiple reactions, some endothermic, some exothermic are actually proceeding in parallel, but the one-step Arrhenius reaction serves as a preliminary model. The viscous dissipation in the melt is given by

$$
\Phi=\mu(T)\left\{2\left[\left(\frac{\partial u}{\partial x}\right)^{2}+\left(\frac{\partial v}{\partial y}\right)^{2}\right]+\left(\frac{\partial v}{\partial x}+\frac{\partial u}{\partial y}\right)^{2}\right\} .
$$

In the presence of both viscous and chemical heating in the melt, the conservation of energy equations in the liquid and solid phases read, in non-dimensional form,

$$
\begin{aligned}
c(T)\left(\frac{\partial T}{\partial t}+u \frac{\partial T}{\partial x}+v \frac{\partial T}{\partial y}\right)= & \frac{1}{\mathrm{Pe}} \nabla \\
& \cdot(\kappa(T) \nabla T) \\
& +\frac{\mathrm{Ec}}{\operatorname{Re}} \Phi+\hat{\Omega} \hat{A}(1-\alpha) \exp \left(-\frac{\hat{E}}{T}\right),
\end{aligned}
$$


and

$$
c\left(T_{\mathrm{sol}}\right) \frac{\partial T_{\mathrm{sol}}}{\partial t}=\frac{1}{\mathrm{Pe}} \nabla \cdot\left(\kappa\left(T_{\mathrm{sol}}\right) \nabla T_{\mathrm{sol}}\right)+\hat{\Omega} \hat{A}(1-\alpha) \exp \left(-\frac{\hat{E}}{T_{\mathrm{sol}}}\right),
$$

respectively. Here $\hat{\Omega}=\Omega /\left(c^{*} \Delta T\right)$ is the non-dimensional heat of reaction, $\Omega$ is the specific heat of the reaction and $c^{*}$ the reference specific heat of the explosive material. The energy equations include the Reynolds number and three further dimensionless groupings: the Péclet number $\mathrm{Pe}=\left(c^{*} \rho v_{\mathrm{w}} l^{*}\right) / \kappa^{*}$, where $\kappa^{*}$ is the reference thermal conductivity; the Eckert number $\mathrm{Ec}=v_{\mathrm{w}}^{2} /\left(c^{*} \Delta T\right)$; and the ratio of heat of the reaction to the sensible heat $\hat{\Omega}=\Omega /\left(c^{*} \Delta T\right)$. The location of the melt front is determined by the Stefan condition, which equates the temperature flux discontinuity across the melt front with the magnitude of the latent heat sink at the phase boundary,

$$
\frac{\partial}{\partial t} x_{f}(y, t)=D\left(-\left.\frac{\partial}{\partial x} T(x, y, t)\right|_{x=x_{f}^{-}}+\left.\frac{\partial}{\partial x} T_{\mathrm{sol}}(x, y, t)\right|_{x=x_{f}^{+}}\right)
$$

where the non-dimensional diffusion coefficient is given by

$$
D=\frac{\mathrm{Ste}}{\mathrm{Pe}}
$$

which is the ratio of the Stefan number to the Péclet number. The Stefan number is the ratio of the sensible heat to the latent heat

$$
\text { Ste }=\frac{c^{*} \Delta T}{L},
$$

where $L$ is the latent heat of the material. The melting temperature is assumed constant, $T=T_{\mathrm{m}}$, and as in [17] we make the equilibrium assumption at the solid-liquid interface, giving the condition

$$
T\left(x_{f}(y, t)\right)=T_{\mathrm{sol}}\left(x_{f}(y, t)\right)=T_{\mathrm{m}} .
$$

In the following we will assume that the wall acts as a insulating boundary, giving

$$
\frac{\partial T}{\partial x}=0 \quad \text { at } \quad x=0
$$

In reality there will be some heat loss to the wall. However, any loss will be negligibly small owing to the very short typical timescale to ignition and comparatively large thermal diffusion time. Note that condition (14) would also be applicable at the midpoint of a symmetric shear layer, as in [17]. In the far-field (i.e. away from the melt front) the temperature of the solid explosive material should decay to the ambient temperature, giving the condition

$$
T_{\text {sol }} \rightarrow \bar{T} \quad \text { as } \quad x \rightarrow \infty
$$

where $\bar{T}=300 \mathrm{~K} / \Delta T$ is the non-dimensional ambient temperature.

Herein we restrict our attention to localised disturbances to the melt front, centred about $y=0$, so that we recover the existing corresponding one-dimensional 
solution as we move away from the disturbance site. This provides the conditions

$$
\frac{\partial T}{\partial y} \rightarrow 0, \quad \frac{\partial T_{\text {sol }}}{\partial y} \rightarrow 0, \quad \text { and } \quad \frac{\partial P}{\partial y} \rightarrow 0 \quad \text { as } \quad y \rightarrow \pm \infty .
$$

The equation for the mass fraction (6) is supplemented by the boundary conditions

$$
\frac{\partial \alpha}{\partial x}=0 \quad \text { at } \quad x=0 \text { and } x=x_{f}, \quad \text { and } \quad \frac{\partial \alpha}{\partial y} \rightarrow 0 \quad \text { as } \quad y \rightarrow \pm \infty
$$

The two-dimensional melt model presented here accounts for the temperature dependence of the specific heat, viscosity and thermal conductivity. We describe the specific heat $c$ using an Einstein relation. This particular functional form has been used by Austin et al. [18] to describe the heat capacity for the $\beta$ phase of HMX, and it is therein assumed that the heat capacity of the liquid phase is identical to that of the solid phase. In the interest of simplicity we make the same assumption here so that the specific heat of liquid HMX is given by

$$
c(T)=\frac{\left(\theta_{1} / T\right)^{2} \exp \left(\theta_{1} / T\right)}{\left(\exp \left(\theta_{1} / T\right)-1\right)^{2}}
$$

where $\theta_{1}=(1000 \mathrm{~K}) / \Delta T$. The temperature dependence of the viscosity $\mu$ is modelled using an Arrhenius law

$$
\mu(T)=\exp \left(\frac{\theta_{2}}{T}-\frac{\theta_{2}}{\theta_{3}}\right)
$$

where $\theta_{2}=(7800 \mathrm{~K}) / \Delta T$ and $\theta_{3}=(800 \mathrm{~K}) / \Delta T$ are experimentally determined constants. For the thermal conductivity $\kappa$ we fit data found in $[19,20]$ using an exponential law

$$
\kappa(T)=\theta_{4}+\theta_{5} \exp \left(-\theta_{6} T\right)
$$

where $\theta_{4}=\left(0.26 \mathrm{~W} \mathrm{~m}^{-1} \mathrm{~K}^{-1}\right) / \kappa^{*}, \theta_{5}=\left(246.08 \mathrm{~W} \mathrm{~m}^{-1} \mathrm{~K}^{-1}\right) / \kappa^{*}$ and $\theta_{6}=$ $\left(0.0145 \mathrm{~K}^{-1}\right) \Delta T$.

The non-dimensional specific heat, viscosity and thermal conductivity are related to their dimensional counterparts through the scalings $c^{*}=1034 \mathrm{~J} \mathrm{~kg}^{-1} \mathrm{~K}^{-1}, \mu^{*}=$ $5.5 \times 10^{-3} \mathrm{~kg} \mathrm{~m}^{-1} \mathrm{~s}^{-1}$ and $\kappa^{*}=0.3884 \mathrm{~W} \mathrm{~m}^{-1} \mathrm{~K}^{-1}$, respectively. For the viscosity we use the values given by Menikoff and Sewell [19], who state that at $800 \mathrm{~K}$ the viscosity of HMX drops to $5.5 \times 10^{-3} \mathrm{~kg} \mathrm{~m}^{-1} \mathrm{~s}^{-1}$, whereas the scaling for the specific heat has been chosen to compare well with previous modelling. The scaling for the thermal conductivity was chosen using the data from [20].

The relevant material properties, their notation, and typical values for HMX, are shown in Table 1. In order to simplify the problem, all material properties are taken to be the same in both the solid and liquid regions. This assumption is consistent with [17], and has been used in other models for HMX [e.g. 18]; the analysis could be extended in a straightforward manner to account for differing material properties. However, in the following lubrication analysis we only treat the liquid layer, and the properties of the solid phase do not appear explicitly at leading-order. As a result, the analysis presented herein would be unchanged. 


\subsection{Lubrication approximation}

After an initial small time period a viscous melt layer will have formed between the moving wall and the solid explosive. The melt layer is expected to be thin in comparison to its length, so we adopt the lubrication approximation (see [21], for example). This essentially reduces the problem to a single-phase Stefan problem in which we are concerned with the temperature evolution in the liquid melt layer. To exploit this expected thinness of the melt layer we adopt the usual scalings

$$
x=\epsilon X, \quad u=\epsilon U, \quad p=\epsilon^{-2} P,
$$

where $\epsilon$ is a small parameter such that $X, U, P$ are $O(1)$ in the thin melt layer. To leading-order we obtain the governing equations for the liquid melt layer

$$
\begin{aligned}
\frac{\partial U}{\partial X}+\frac{\partial v}{\partial y} & =0 \\
\frac{\partial P}{\partial X} & =0 \\
-\frac{\partial P}{\partial y}+\frac{\partial}{\partial X}\left(\mu(T) \frac{\partial v}{\partial X}\right) & =0
\end{aligned}
$$

which are based on the usual assumption that both $\epsilon \ll 1$ and $\epsilon^{2} \mathrm{Re} \ll 1$. Equations $(22)-(24)$ are to be solved subject to no-slip boundary conditions on the wall and melt front, where the liquid melt layer will be in contact with the unmelted solid explosive. Since we are restricting our attention to localised disturbances we expect that far from the site of the two-dimensional disturbance the solution will resemble the one-dimensional solution, and that the pressure will be a function of time only.

Substitution of the lubrication layer scalings (21) into the energy equation for the melt layer (8) provides

$$
\begin{aligned}
c(T)\left(\frac{\partial T}{\partial t}+U \frac{\partial T}{\partial X}+v \frac{\partial T}{\partial y}\right) & =\frac{1}{\mathrm{Pe}} \epsilon^{-2}\left(\frac{\partial \kappa(T)}{\partial X} \frac{\partial T}{\partial X}+\kappa(T) \frac{\partial^{2} T}{\partial X^{2}}\right) \\
+ & \frac{1}{\operatorname{Pe}}\left(\frac{\partial \kappa(T)}{\partial y} \frac{\partial Y}{\partial y}+\kappa(T) \frac{\partial^{2} T}{\partial y^{2}}\right)+\frac{\operatorname{Ec}}{\operatorname{Re}} \Phi+\hat{\Omega} \hat{A}(1-\alpha) \exp \left(-\frac{\hat{E}}{T}\right),
\end{aligned}
$$

where the expansion of the mechanical dissipation is given by

$$
\Phi=\mu(T)\left\{2\left[\left(\frac{\partial U}{\partial X}\right)^{2}+\left(\frac{\partial v}{\partial y}\right)^{2}\right]+\epsilon^{-2}\left(\frac{\partial v}{\partial X}\right)^{2}+\epsilon^{2}\left(\frac{\partial u}{\partial y}\right)^{2}+2 \frac{\partial v}{\partial X} \frac{\partial U}{\partial y}\right\}
$$

The energy equation (25) is to be solved subject to the boundary conditions

$$
\frac{\partial T}{\partial X}=0 \text { at } X=0, \quad T=T_{\mathrm{m}} \text { at } X=X_{f}(y, t), \quad \frac{\partial T}{\partial y} \rightarrow 0 \text { as } y \rightarrow \pm \infty .
$$

The small parameter $\epsilon$ is fixed to obtain a dominant balance between convection, diffusion and dissipation terms in the melt layer. This amounts to ensuring the 
horizontal diffusion term in the energy equation $(25)$ is $O(1)$, so that

$$
\epsilon^{2}=\frac{1}{\mathrm{Pe}}
$$

For the material properties used in this study we find that the Péclet number is in the range $10^{3}<\mathrm{Pe}<10^{6}$, depending on wall speed $v_{\mathrm{w}}$, so that $\epsilon$ is indeed small.

We retain the leading-order terms, along with the reaction term which is initially small but grows rapidly as the temperature increases. The leading-order energy equation in the melt layer now reads

$$
\begin{aligned}
c(T)\left(\frac{\partial T}{\partial t}+U \frac{\partial T}{\partial X}+v \frac{\partial T}{\partial y}\right)= & \frac{\partial \kappa(T)}{\partial X} \frac{\partial T}{\partial X}+\kappa(T) \frac{\partial^{2} T}{\partial X^{2}} \\
& +\operatorname{Ec} \operatorname{Pr} \Phi+\hat{\Omega} \hat{A}(1-\alpha) \exp \left(-\frac{\hat{E}}{T}\right),
\end{aligned}
$$

with $\Phi=\mu(T)(\partial v / \partial X)^{2}$. Here we have introduced the Prandtl number, defined as $\operatorname{Pr}=\left(c^{*} \mu^{*}\right) / \kappa^{*}$. Note that for heat transfer the Péclet number can be written as the product of the Reynolds number and the Prandtl number: Pe $=\operatorname{Re} \operatorname{Pr}$. The rescaled Stefan condition (10) reads

$$
\epsilon \frac{\partial}{\partial t} X_{f}(y, t)=D\left(-\left.\epsilon^{-1} \frac{\partial T}{\partial X}\right|_{X=X_{f}^{-}}+\left.\epsilon^{-1} \frac{\partial}{\partial X}\left(\epsilon T_{\mathrm{sol}}^{\prime}\left(X=X_{f}\right)+O\left(\epsilon^{2}\right)\right)\right|_{X=X_{f}^{+}}\right)
$$

where the solid temperature has been expanded about the melt front so that the size of its derivative may be properly determined with respect to $\epsilon$. For the material properties quoted for HMX, and taking a typical temperature difference to be $\Delta T \sim O(100 \mathrm{~K})$, it is found that the Stefan number is typically $O(1)$. This makes the diffusion coefficient $D \sim O\left(\epsilon^{2}\right)$ so that the leading-order Stefan condition is given by

$$
\frac{\partial X_{f}}{\partial t}=- \text { Ste }\left.\frac{\partial T}{\partial X}\right|_{X=X_{f}^{-}}
$$

\subsection{Front-fixing transformation}

In Section 3.2 the governing equations in the liquid region $(22)-(24),(? ?)$ and (31), along with the Arrhenius equation (6) are to be solved numerically. In order to facilitate this we introduce the front-fixed co-ordinate $\xi=X / X_{f}$. This significantly simplifies the numerical treatment, and formally recovers a fixed grid. The $3 \times 3$ Jacobian transformation matrix reads

$$
\boldsymbol{J}=\frac{\partial(\xi, y, t)}{\partial(X, y, t)}=\left(\begin{array}{cccc}
\frac{1}{X_{f}} & 0 & 0 \\
-\frac{\partial X_{f}}{\partial y} \frac{\xi}{X_{f}} & 1 & 0 \\
-\frac{\partial X_{f}}{\partial t} \frac{\xi}{X_{f}} & 0 & 1
\end{array}\right)
$$

Under this coordinate transformation the moving phase boundary is located exactly at $\xi=1$. The governing equations for the melt front take the following form in the 
front-fixing coordinates:

$$
\begin{aligned}
\frac{1}{X_{f}} \frac{\partial U}{\partial \xi}-\frac{\partial X_{f}}{\partial y} \frac{\xi}{X_{f}} \frac{\partial v}{\partial \xi}+\frac{\partial v}{\partial y} & =0 \\
\frac{1}{X_{f}} \frac{\partial P}{\partial \xi} & =0 \\
\frac{\partial X_{f}}{\partial y} \frac{\xi}{X_{f}} \frac{\partial P}{\partial \xi}-\frac{\partial P}{\partial y}+\frac{1}{X_{f}^{2}} \frac{\partial}{\partial \xi}\left(\mu(T) \frac{\partial v}{\partial \xi}\right) & =0 \\
c(T)\left(\frac{\partial T}{\partial t}+\frac{1}{X_{f}}\left(U-\frac{\partial X_{f}}{\partial t} \xi\right.\right. & \left.\left.-v \frac{\partial X_{f}}{\partial y} \xi\right) \frac{\partial T}{\partial \xi}+v \frac{\partial T}{\partial y}\right) \\
=\frac{1}{X_{f}^{2}}\left(\frac{\partial \kappa(T)}{\partial \xi} \frac{\partial T}{\partial \xi}+\kappa(T) \frac{\partial^{2} T}{\partial \xi^{2}}\right)+\operatorname{Ec} \operatorname{Pr} \Phi & +\hat{\Omega} \hat{A}(1-\alpha) \exp \left(-\frac{\hat{E}}{T}\right), \\
\frac{\partial X_{f}}{\partial t}+\left.\operatorname{Ste} \frac{1}{X_{f}} \frac{\partial T}{\partial \xi}\right|_{\xi=1^{-}} & =0, \\
\frac{\partial \alpha}{\partial t}+\frac{1}{X_{f}}\left(U-\frac{\partial X_{f}}{\partial t} \xi-v \frac{\partial X_{f}}{\partial y} \xi\right) \frac{\partial \alpha}{\partial \xi}+v \frac{\partial \alpha}{\partial y} & =\hat{A}(1-\alpha) \exp \left(-\frac{\partial v}{\partial \xi}\right)^{2}
\end{aligned}
$$

where velocity components $U, v$, temperature $T$, mass fraction $\alpha$ and are all functions of $\xi, y, t$, and pressure $P$ and melt front location $X_{f}$ are functions of $y$ and $t$ only. This formulation of the problem will be used to obtain asymptotic results in Sections 3.1 and 4 .

In the preceding analysis we have derived a two-dimensional model for the melting of HMX. The introduction of the lubrication approximation has reduced the problem to a single-phase Stefan problem, in which the melt front only propagates in the direction perpendicular to the wall located at $\xi=0(X=0)$. The solid region of unmelted explosive is now considered to be of infinite expanse, occupying the region $\xi>1\left(X>X_{f}\right)$, and is held fixed at the melting temperature $T_{\mathrm{m}}$. In the absence of any $y$ dependence the analysis in this section may be viewed as a formal derivation of the ad-hoc one-dimensional model for a thin shear-melted viscous layer presented in [17]. Equivalently, the model presented in [17] may be recovered by considering early time solutions of the two-dimensional model (see Appendix A).

\section{Shear melt model: constant material properties}

In order to simplify the analysis, and to compare results with [17], the specific heat, viscosity and thermal conductivity are treated as constant in this section, taking dimensional values $\tilde{c}=989.25 \mathrm{~J} \mathrm{~kg}^{-1} \mathrm{~K}^{-1}, \tilde{\mu}=4.6 \times 10^{-2} \mathrm{~kg} \mathrm{~m}^{-1} \mathrm{~s}^{-1}$ and $\tilde{\kappa}=0.404 \mathrm{~W} \mathrm{~m}^{-1} \mathrm{~K}^{-1}$, respectively. Since the material properties are assumed independent of temperature, the functions (18) - (20) are constant $c(T)=\mu(T)=$ $\kappa(T)=1$. This assumption will be relaxed in Section 4, where the effects of temperature dependent material properties will be investigated, and are shown to make only a minor qualitative difference to the hot spot generation mechanisms discussed 
here.

For constant material properties, the mechanical and thermal equations decouple and explicit expressions may be found for the velocity components. Equation (34) immediately requires that the pressure in the lubrication layer is independent of $\xi$, i.e. $P=P(y, t)$. Integrating (35) directly with respect to $\xi$ and applying boundary conditions we obtain an expression for the vertical velocity component

$$
v(\xi, y, t)=\frac{1}{2} X_{f}^{2} \frac{\partial P}{\partial y} \xi(\xi-1)-(1-\xi)
$$

The horizontal velocity component may then be calculated by integration of the continuity equation (33) a distance $\xi$ across the layer

$$
U(\xi, y, t)=\frac{1}{2} \frac{\partial X_{f}}{\partial y} \xi^{2}+\frac{1}{12}\left(3 X_{f}^{2} \frac{\partial X_{f}}{\partial y} \frac{\partial P}{\partial y} \xi^{2}+6 X_{f}^{3} \frac{\partial^{2} P}{\partial y^{2}}\left(\frac{\xi^{2}}{2}-\frac{\xi^{3}}{3}\right)\right)
$$

Application of the no-slip boundary conditions then provides a condition relating the position of the melt front and the pressure gradient

$$
\frac{\partial X_{f}}{\partial y}+\frac{\partial}{\partial y}\left(\frac{X_{f}^{3}}{6} \frac{\partial P}{\partial y}\right)=0
$$

In Section 3.2 the above expressions will be substituted into the energy equation, which is solved numerically subject to the Stefan condition. The simplified system of equations that result from assuming constant material properties allows consideration of larger departures from a uniform melt front in our numerical analysis.

\subsection{Asymptotic analysis for small disturbances to the melt front}

In this section we consider small deviations from a flat melt front $X_{f 0}=X_{f 0}(t)$ for both reactive and non-reactive materials in order to assess the stability of the melt front in the presence and absence of any chemical reaction. The problem is linearised about $X_{f 0}$, and formulated in terms of the leading-order front-fixed coordinate $\zeta=X / X_{f 0}$ instead of $\xi=X / X_{f}$. It is demonstrated that the melt front is linearly stable to small disturbances in the absence of the chemical reaction, but linearly unstable upon inclusion of the Arrhenius source term. We look for a solution in terms of a perturbation series, writing

$$
\begin{aligned}
X_{f} & =X_{f 0}(t)+\delta X_{f 1}(t) S(y)+O\left(\delta^{2}\right), \\
P(y, t) & =P_{0}(t)+\delta P_{1}(y, t)+O\left(\delta^{2}\right) \\
{[U, v, T, \alpha](\zeta, y, t) } & =\left[U_{0}, v_{0}, T_{0}, \alpha_{0}\right](\zeta, t)+\delta\left[U_{1}, v_{1}, T_{1}, \alpha_{1}\right](\zeta, y, t)+O\left(\delta^{2}\right),
\end{aligned}
$$

where $0<\delta \ll 1$ and $S(y)$ is a given function describing the shape of the localised disturbance in the melt front. Note that since we are only considering localised disturbances we require that $S \rightarrow 0$ as $y \rightarrow \pm \infty$. We further stipulate that this shape must be slowly varying in the $y$ direction so that its derivatives may be neglected to a first approximation.

The expansions (43) - (45) are substituted into the governing equations (33) (39) and terms with like powers of $\delta$ are collected to form a series of problems to 
be solved. At leading-order we have the problem

$$
\begin{aligned}
\frac{1}{X_{f 0}} \frac{\partial U_{0}}{\partial \zeta} & =0 \\
\frac{1}{X_{f 0}^{2}} \frac{\partial^{2} v_{0}}{\partial \zeta^{2}} & =0 \\
\frac{\partial T_{0}}{\partial t}+\frac{1}{X_{f 0}}\left(U_{0}-\frac{\partial X_{f 0}}{\partial t} \zeta\right) \frac{\partial T_{0}}{\partial \zeta} & =\frac{1}{X_{f 0}^{2}} \frac{\partial^{2} T_{0}}{\partial \zeta^{2}}+\operatorname{Ec} \operatorname{Pr} \Phi_{0}+\hat{\Omega} \frac{\partial \alpha_{0}}{\partial t}, \\
\Phi_{0} & =\frac{1}{X_{f 0}^{2}}\left(\frac{\partial v_{0}}{\partial \zeta}\right)^{2} \\
\frac{\partial X_{f 0}}{\partial t} & =-\left.\operatorname{Ste} \frac{1}{X_{f 0}} \frac{\partial T_{0}}{\partial \zeta}\right|_{\zeta=1^{-}}, \\
\frac{\partial \alpha_{0}}{\partial t}+\frac{1}{X_{f 0}}\left(U_{0}-\frac{\partial X_{f 0}}{\partial t} \zeta\right) \frac{\partial \alpha_{0}}{\partial \zeta} & =\hat{A}\left(1-\alpha_{0}\right) \exp \left(-\frac{\hat{E}}{T_{0}}\right)
\end{aligned}
$$

with boundary conditions

$$
\begin{aligned}
& U_{0}=0, \quad v_{0}=-1, \quad \frac{\partial T_{0}}{\partial \zeta}=0, \quad \frac{\partial \alpha_{0}}{\partial \zeta}=0 \quad \text { on } \zeta=0, \\
& U_{0}=0, \quad v_{0}=0, \quad T_{0}=T_{\mathrm{m}}, \quad \frac{\partial \alpha_{0}}{\partial \zeta}=0 \quad \text { on } \zeta=1 .
\end{aligned}
$$

At $O(\delta)$ we have the problem

$$
\begin{aligned}
\frac{1}{X_{f 0}} \frac{\partial U_{1}}{\partial \zeta}+\frac{\partial v_{1}}{\partial y} & =0 \\
-\frac{\partial P_{1}}{\partial y}+\frac{1}{X_{f 0}^{2}} \frac{\partial^{2} v_{1}}{\partial \zeta^{2}} & =0 \\
\frac{\partial T_{1}}{\partial t}+\frac{1}{X_{f 0}}\left(U_{0}-\frac{\partial X_{f 0}}{\partial t} \zeta\right) \frac{\partial T_{1}}{\partial \zeta}+\frac{1}{X_{f 0}}\left(U_{1}-\frac{\partial X_{f 1}}{\partial t} S \zeta\right) \frac{\partial T_{0}}{\partial \zeta}+v_{0} \frac{\partial T_{1}}{\partial y} & =\frac{1}{X_{f 0}^{2}} \frac{\partial^{2} T_{1}}{\partial \zeta^{2}}+\operatorname{Ec} \operatorname{Pr} \Phi_{1}+\hat{\Omega} \frac{\partial \alpha_{1}}{\partial t} \\
\frac{\partial X_{f 1}}{\partial t} & =-\frac{2}{X_{f 0}^{2}} \frac{\partial v_{0}}{\partial \zeta} \frac{\partial v_{1}}{\partial \zeta}, \\
\frac{\partial \alpha_{1}}{\partial t}+\frac{1}{X_{f 0}}\left(U_{0}-\frac{X_{f 1}}{\partial t} \zeta\right) & \frac{\partial \alpha_{1}}{\partial \zeta}+\left.\frac{1}{X_{f 0}^{2}}\left(T_{0}+\frac{1}{X_{f 0}} \frac{\partial T_{1}}{\partial \zeta}\right)\right|_{\zeta=1^{-}} \\
& =\hat{A}\left[\left(1-\alpha_{0}\right) \frac{\partial X_{f 1}}{T_{0}^{2}} T_{1}-\alpha_{1}\right] \frac{\partial \alpha_{0}}{\partial \zeta}+v_{0} \frac{\partial \alpha_{1}}{\partial y}
\end{aligned}
$$


with boundary conditions

$$
\begin{gathered}
U_{1}=0, \quad v_{1}=0, \quad \frac{\partial T_{1}}{\partial \zeta}=0, \quad \frac{\partial \alpha_{1}}{\partial \zeta}=0 \quad \text { on } \zeta=0 \\
\frac{X_{f 1}}{X_{f 0}} \frac{\partial U_{0}}{\partial \zeta}+U_{1}=0, \quad \frac{X_{f 1}}{X_{f 0}} \frac{\partial v_{0}}{\partial \zeta}+v_{1}=0, \quad \frac{X_{f 1}}{X_{f 0}} \frac{\partial T_{0}}{\partial \zeta}+T_{1}=0 \\
\frac{X_{f 1}}{X_{f 0}} \frac{\partial \alpha_{0}}{\partial \zeta}+\alpha_{1}=0 \quad \text { on } \zeta=1 .
\end{gathered}
$$

It is found that $U_{1} \sim S^{\prime}(y)$, so the horizontal velocity is neglected up to $O(\delta)$ in this analysis.

\subsubsection{Linearised model: non-reactive material}

Here we consider the heating solely due to mechanical dissipation in the melt layer. Naturally, we take $\hat{A}=0$ since we are considering non-reactive materials. The leading-order problem is the same as that found in [17] and has an analytical solution, whereas the first-order problem is solved numerically using an iterative Crank-Nicolson scheme.

It is easily verified that a steady state velocity profile satisfies the equations (46) and (47) for the leading-order velocity components

$$
U_{0}(\zeta, t)=0, \quad v_{0}(\zeta, t)=\zeta-1
$$

which, by equation (49), predicts uniform leading-order dissipative heating $\Phi_{0}(t)=$ $1 / X_{f 0}^{2}$. The same solution may be found by substitution of the expansions into equations (40) - (42). In the absence of any chemical reaction, the leading order equations admit a self-similar analytical solution which has parabolic advancement of the melt front $X_{f 0}=q \sqrt{t}$, with propagation parameter $q$. As in [17], the leadingorder temperature may be written as a sum of of two functions

$$
T_{0}(\zeta, t)=f(\zeta, t)+h(\zeta)
$$

The function $h(\zeta)$ satisfies the time-independent form of (48), along with the appropriate boundary conditions, and is given by

$$
\begin{aligned}
h(\zeta)=T_{\mathrm{m}}+ & \operatorname{Ec} \operatorname{Pr} \frac{\sqrt{\pi}}{2 u}\left(\operatorname{erf}(\sqrt{u}) \int_{0}^{\sqrt{u}} e^{q^{2}} \mathrm{~d} q-\int_{0}^{\sqrt{u}} \operatorname{erf}(q) e^{q^{2}} \mathrm{~d} q\right) \\
& -\operatorname{Ec} \operatorname{Pr} \int_{0}^{\zeta} e^{-u \zeta_{2}^{2}} \int_{0}^{\zeta_{2}} e^{u \zeta_{1}^{2}} \mathrm{~d} \zeta_{1} \mathrm{~d} \zeta_{2},
\end{aligned}
$$

with the propagation parameter $u=q^{2} / 4$ satisfying

$$
2 u=\operatorname{Ste} \operatorname{Ec} \operatorname{Pr} \frac{e^{-u}}{\sqrt{u}} \int_{0}^{\sqrt{u}} e^{q^{2}} \mathrm{~d} q .
$$


The function $f(\zeta, t)$ satisfies the equation

$$
q^{2} t \frac{\partial f}{\partial t}=\frac{\partial^{2} f}{\partial \zeta^{2}}+\frac{q^{2}}{2} \zeta \frac{\partial f}{\partial \zeta}
$$

and must have vanishing derivative at $\zeta=0$. We also require $f(1, t)=0$ so that the temperature is equal to the melt temperature $T_{\mathrm{m}}$ at the melt front. It can be shown (see [17]) that the function $f(\zeta, t)$ has the following form:

$$
f(\zeta, t) \propto\left(\frac{t_{0}}{t}\right)^{\beta^{2} / q^{2}}{ }_{1} F_{1}\left(\frac{\beta^{2}}{q^{2}}, \frac{1}{2},-\frac{q^{2}}{4} \zeta^{2}\right),
$$

where $t_{0}$ is the initial time, ${ }_{1} F_{1}$ is a confluent hypergeometric function [22], and $\beta$ is a constant which is fixed by ensuring $f(\zeta, t)$ vanishes at $\zeta=1$.

Figure 2 shows the melt front correction $X_{f 1}$ and melt front propagation speed $\dot{X}_{f 1}$ both as a function of time. It is found that small spatial variations in the melt front are linearly stable, always decaying back to the one-dimensional case for a non-reactive material. We observe that the propagation speed decays very rapidly, and we soon return to a solution close to the one-dimensional solution.

Figure 3 (a) shows the leading-order velocity, i.e. the linear profile, along with the velocity computed up to $O(\delta)$. We observe a small departure from the linear profile, which in turn creates some spatial variation in the mechanical heating across the melt layer, as seen in Figure 3 (b) which depicts the first-order correction to the dissipation at a series of increasing times. The correction to the mechanical dissipation may be calculated in terms of the melt front correction $X_{f 1}$ as

$$
\Phi_{1}(\zeta, y, t)=4 \frac{X_{f 1}}{X_{f 0}}(1-3 \zeta) S(y)
$$

which has a root at $\zeta=1 / 3$, so that we always observe an area of increased dissipative heating along with an accompanying area of decreased heating. The locations of these so called "mechanical hot/cool spots" will depend on the sign of the shape function $S(y)$. Irrespective of the selected shape, Figure 3 shows clear localisation of mechanical dissipation which may serve as a source for hot spot generation.

The results of the perturbation analysis can be investigated for different melt front shapes and the key results are discussed here. For initial conditions which solely act to widen the melt layer $(S(y)>0)$ we find increased dissipative heating adjacent to the moving wall and decreased heating on the opposite side of the melt layer, near the solid explosive. Localised dissipation is also witnessed in computations using initial conditions which perturb the melt front towards the wall $(S(y)<0)$. However, this heating happens adjacent to the unmelted solid explosive material, with an accompanying region close to the wall in which the dissipation is lower. In shapes which provide both positive and negative disturbances both heating mechanisms are present - we observe increased dissipative heating both near the wall where the melt layer widens and near the solid explosive where the melt layer narrows.

The locations of the mechanical hot, and accompanying cool, spots may be understood by consideration of the dissipation term near the sliding wall and melt front. Upon substitution of the analytical solution for the vertical velocity compo- 
nent (40) into the dissipation (37) we find

$$
\begin{aligned}
& \Phi \sim \frac{1}{X_{f}^{2}}\left(1+\frac{X_{f}^{4}}{4}\left(\frac{\partial P}{\partial y}\right)^{2}\right)-\frac{\partial P}{\partial y} \quad \text { near } \xi=0(X=0), \\
& \Phi \sim \frac{1}{X_{f}^{2}}\left(1+\frac{X_{f}^{4}}{4}\left(\frac{\partial P}{\partial y}\right)^{2}\right)+\frac{\partial P}{\partial y} \quad \text { near } \xi=1\left(X=X_{f}\right) .
\end{aligned}
$$

We observe that the final correction term to the heating depends on the sign of the pressure gradient, which is negative for disturbances away from the wall and positive for disturbances towards the wall. The above considerations also explain why we always observe an accompanying mechanical cool spot on the opposite side of the melt layer to each hot spot.

Clearly the solution for the non-reactive material cannot make any predictions regarding time to thermal runaway or locations of increased chemical heating. However, a large temperature increase is required to start the chemical reaction, so the localisation of heating due to mechanical dissipation is an excellent indicator of possible hot spot locations.

\subsubsection{Linearised model: reactive material}

We now account for self-heating due to exothermic reaction in the melt layer. The non-dimensional rate constant $\hat{A}=t^{*} A$ is no longer taken to be zero, and we include an Arrhenius heat source, described by equations (51) and (59), in our analysis to account for the chemical reaction. In this case, both the leading- and first-order equations are solved numerically. Since the material properties are considered to be constant, the velocity profile remains unchanged, as does the mechanical heating in the layer. However, the heat generated by the chemical reactions affects the propagation of the melt front. Figure 4 shows the first-order correction to the melt front $X_{f 1}$ and its propagation speed $\dot{X}_{f 1}$. In contrast to the non-reactive results we find that the perturbation begins to grow in time, i.e. the melt front is found to be linearly unstable upon inclusion of the Arrhenius source term. This destabilising effect introduced by the chemical reaction may lead to the growth of local disturbances to the melt front, which in turn may lead to the formation of more violent hot spots.

\section{$3.2 \quad$ Numerical results for a reactive material}

We now return to the full problem described by (33) - (39), including the Arrhenius source term. The model equations for the liquid region in the front-fixing coordinates are solved numerically using an iterative Crank-Nicolson scheme, subject to the boundary conditions which are the front-fixed counterparts of conditions (27), which read

$$
\frac{1}{X_{f}} \frac{\partial T}{\partial \xi}=0 \text { at } \xi=0, \quad T=T_{\mathrm{m}} \text { at } \xi=1, \quad \frac{\partial T}{\partial y} \rightarrow 0 \text { as } y \rightarrow \pm \infty,
$$

where we have used the fact that we are studying localised shapes $(S \rightarrow 0$ as $y \rightarrow \pm \infty)$ to remove the terms arising from the coordinate transformation in the final condition. We solve the problem on an $N_{\xi} \times N_{y}$ grid, with subscripts $i=1, \ldots, N_{\xi}$ and $j=1, \ldots, N_{y}$ indexing the position and superscript $k$ denoting 
the time step. The convective terms were discretised using a two-point centred difference and the second order diffusive term was discretised using a three point centred difference, both of which are averaged over the current and subsequent time steps (see Appendix B). For the time derivative a second order centred difference is used to evaluate the derivative at the point $\left(x_{i}, y_{j}, t_{k+1 / 2}\right)$. The nonlinear terms were evaluated at the mid-level, in-keeping with the rest of the scheme. This gives overall truncation error $O\left(\Delta \xi^{2}, \Delta y^{2}, \Delta t^{2}\right)$, where $\Delta \xi, \Delta y$ and $\Delta t$ are the grid and time spacing, respectively. Typically a grid spacing of $\Delta \xi=0.02$ and $\Delta y=0.06$ was used. For the time-stepping an adaptive scheme was used allowing for larger steps to be taken in the early stage of the simulation before the onset of rapid reaction.

The method uses an initial condition provided by the small time solution given in Appendix A and proceeds in the following way:

(1) The current iterate of the temperature is computed using the discrete form of the energy equation (36) using the current melt front location.

(2) The current melt front location is updated using the Stefan condition (38) with the current temperature iterate.

(3) The current iterate of the pressure gradient is updated using (42).

This process is iterated until the melt front location converges. In practice it is found that 2 or 3 iterations is normally sufficient to meet a convergence threshold of $10^{-6}$ in the relative change in the melt front location.

To obtain a suitable initial condition for computations an initial time $t_{0}$ is selected, and the time-independent part of the analytical one-dimensional solution for non-reactive materials (64) is applied at each $y$ station in the finite difference scheme. This approach is consistent with the assumption that the melt layer has already formed before being perturbed. The one-dimensional solution also determines the thickness of the melt layer at the initial time $X_{f}\left(t_{0}\right)=q \sqrt{t_{0}}$. The liquid speed at the wall will remain equal to the sliding speed for melt layers thicker than approximately $10 \mathrm{~nm}$ [17], so in order to apply the no-slip boundary condition used in this analysis we must select an initial time $t_{0}$ which provides an initial dimensional melt layer thickness of at least $10 \mathrm{~nm}$. Typically an initial time of $10 \mathrm{~ns}$ was used. A selection of different initial times were taken, and the choice of $t_{0}$ was shown to have a negligible effect on the results below. At time $t=t_{0}^{+}$the uniform melt front is perturbed via a shape function $S(y)$ so that it is now a function of space and time $X_{f}=X_{f}\left(y, t_{0}^{+}\right)$. The numerical scheme is then used to evolve the temperature field forward in time subject to the Stefan condition.

Results are presented for a sample of HMX subject to a uniform wall speed $v_{\mathrm{w}}$. A number of melt front shapes were studied. Here we draw comparison between two initial shapes which describe the $y$ dependence of the initial melt front $X_{f}\left(y, t_{0}^{+}\right)$, and are parametrised by $\delta: S_{1}(\delta, y)=1+(\delta / 2) e^{-y^{2}}$ and $S_{2}(\delta, y)=1+\delta e^{-2 y^{2}} \sin (\pi y)$. For shape $S_{1}$ positive values of $\delta$ correspond to a local widening of the melt layer, whereas negative values of $\delta$ correspond to a local narrowing of the melt layer. Shape $S_{2}$ describes a localised sinusoidal perturbation the melt front shape. In the following the time to ignition is defined as the time at which the peak temperature exceeds $1000 \mathrm{~K}$.

Figure 5 compares the non-dimensional propagation constant $q$ calculated from equation (65), with the numerically computed propagation constant for a flat melt front profile, i.e. $X_{f}=X_{f}(t)$. The agreement is shown to be good, with numerical and analytical values agreeing to within one percent, for grid sizes $\Delta \xi=0.05$, $\Delta y=0.05$ and timestep $\Delta t=10^{-4}$. It is found in practice that the time scale $t^{*}$ 
should decrease logarithmically from $10^{-4} \mathrm{~s}$ to $10^{-7} \mathrm{~s}$ over the range of wall speeds $v_{\mathrm{w}}=50-80 \mathrm{~m} \mathrm{~s}^{-1}$. Figure 6 shows the time to ignition and maximum melt width for a sample of HMX with a uniform melt layer, which corresponds exactly to the one-dimensional model. As in [17], we observe an order of magnitude decrease in the ignition time and melt width as the wall speed is increased.

Somewhat counterintuitively, we find that making the melt layer locally wider, as in $S_{1}$ with $\delta>0$, appears to be the most violent initial condition in the sense of decreasing time to runaway. It is found that the heating due to mechanical dissipation is greatest adjacent to the wall and opposite from the imposed disturbance in the melt front, see Figure 7 (a). The location of the hot spot is due to the sign of the pressure gradient induced by the perturbation to the melt front, see equation (70). The temperature rise here is sufficient to kickstart a local reaction, causing the temperature to rise rapidly. This has the resultant effect of causing the disturbance to grow in magnitude, causing further temperature localisation. Thus the overall time to runaway is reduced when compared with the one-dimensional case. The high contribution of heating due to reaction is clearly depicted in Figure 7.

The evolution of the melt front, along with the temperature profiles across the width of the melt layer, is shown in Figure 8. Looking at the temperature across the melt width, we observe a temperature rise of over $200 \mathrm{~K}$ near the reaction site. However, away from the reaction site we see that the temperature profile across the melt width has remained almost unchanged throughout the duration of the computation.

As in the linearised case in Section 3.1, it is found that upon reintroduction of the chemical reaction the melt front becomes unstable to small perturbations. This can be observed in Figure 8 (b) which depicts the perturbation from the uniform melt layer at a series of increasing times. We see that initially the disturbance decays, as in the non-reactive material, but towards the end of the computation the disturbance begins to grow again, albeit only by a small amount by the time the ignition threshold was reached. In particular this result demonstrates the ability of the chemical reaction to turn what was a stable system into an unstable one.

In shapes which cause the melt layer to be locally more narrow (i.e. $S_{1}$ with $\delta<0$ ) we find the dissipation to be greatest at the melt front, see Figure 9 (a) and equation (69). The additional temperature increase near the unmelted explosive material causes the melt front to propagate more quickly at the disturbance site, so that the melt layer flattens, returning to a uniform width. We find that the hot spot generated by the narrowing of the melt layer is quenched to the melt temperature $T_{\mathrm{m}}$ by the unmelted solid explosive, so does not have the effect of decreasing the time to runaway. We conclude that a hot spot located on the melt front has little or no effect on the time to runaway. Indeed, we observe that the chemical reaction finally takes off on the wall, but away from the site of the localised disturbance owing to the corresponding mechanical cool spot, see Figure 9 (b). This is further illustrated in Figure 10, where we observe that the temperature increase is less near the disturbance (Figure 10 (c)) than it is away from the disturbance (Figure $10(\mathrm{~d})$ ). In this case the reaction takes longer to kick off, and we observe a noticeable temperature increase throughout the melt layer over the duration of the computation. Since the reaction occurs on the wall away from the perturbation, the bulk of the melt layer propagates ahead of the narrow area, resulting in the apparent growth of the perturbation in the later stages of melting.

Figures 11 and 12 show similar results for the localised sinusoidal disturbance $S_{2}$, which combines both effects seen in shape $S_{1}$ with positive and negative $\delta$. As one might expect for such a small disturbance, we see no interaction between the 
two heating mechanisms and we observe two hot spots.

The time to ignition as a function of $\delta$ for both shapes $S_{1}(\delta, y)$ and $S_{2}(\delta, y)$ is shown in Figure 13. The quenching behaviour is clearly demonstrated; for perturbations which only serve to narrow the melt width (shape $S_{1}, \delta<0$ ), we see that the ignition time is unchanged from that of a uniform melt layer, whereas for positive disturbances $(\delta>0)$ the ignition time decreases as $\delta$ increases.

\section{Shear melt model: temperature-dependent material properties}

In this section we consider the effects of allowing the specific heat, viscosity and thermal conductivity to vary with temperature. When the assumption of constant material properties is relaxed the lubrication equations cannot be integrated directly, so we instead adopt an asymptotic approach. It is shown that the hot spot mechanisms discussed in Section 3 are still present when the assumption of constant material properties is relaxed. Full numerical solution of the coupled equations (33) - (39) would involve iteration between the mechanical and thermal equations and be more complicated than the analysis presented in Section 3.2. Since the results are found to be qualitatively similar in both the constant and non-constant material properties model, only the asymptotic results are presented here, and not the full numerical solution.

As in Section 3.1 we look for a solution in terms of a perturbation series as given by $(43)-(45)$, with the additional expansions for the specific heat, viscosity and thermal conductivity

$$
[c, \mu, \kappa](\zeta, y, t)=\left[c_{0}, \mu_{0}, \kappa_{0}\right](\zeta, t)+\delta\left[c_{1}, \mu_{1}, \kappa_{1}\right](\zeta, y, t)+O\left(\delta^{2}\right),
$$

where $0<\delta \ll \epsilon \ll 1$ is a small parameter characterising the size of the twodimensional disturbance. As in the previous sections, we stipulate that this shape must decay in the far field, so that our one-dimensional solution is applicable as $y \rightarrow \pm \infty$. It is recognised that the specific heat and viscosity may be expressed in terms of the temperature expansion. However, the expansions (72) provide a useful notational convenience. The expansions (43) - (45) and (72) are to be substituted into the governing equations (33) - (39).

First we present the leading-order solutions, demonstrating a departure from the linearised velocity profile used by Starobin and Dienes [17] and the resulting localisation in the mechanical heating across the layer. The leading-order solutions correspond to the one-dimensional case, that is an unperturbed melt front of uniform width. Comparison of the leading-order results with the one-dimensional model presented in [17] allows for the identification of heating mechanisms due solely to modelling the temperature dependence of material properties. We then present an example two-dimensional solution computed up to $O(\delta)$ in the analysis and observe that the localisation mechanisms discussed in the previous section persist when we relax the assumption of constant specific heat, viscosity and thermal conductivity. The leading and first-order problems were solved using the front-fixed coordinate system and iterative Crank-Nicolson scheme presented in Section 3.2. In the interest of brevity we only present a single two-dimensional solution to illustrate the effects of temperature dependent material properties, but similar conclusions hold for the other melt front shapes discussed in this paper.

Figure 14 shows the leading-order specific heat, viscosity and thermal conductivity across the melt layer at a series of increasing times. Figure 14(d) shows the temperature dependence of each of the three properties, scaled by their values at 
a reference temperature of $750 \mathrm{~K}$. We observe that the specific heat increases with temperature, with the shape of the plot looking much like a typical temperature profile across the layer. Conversely, both the viscosity and thermal conductivity decrease with temperature, thus taking their smallest values adjacent to the moving wall where the temperature is greatest. Interestingly we see very little change in the viscosity and specific heat as functions of time, with all snapshots virtually coinciding. The temperature profile used as an initial condition is such that we already observe a dramatic decrease in the viscosity and specific heat across the melt layer, and the local changes in temperature have relatively little effect on both of these properties as time proceeds. Note that since the leading-order equations reduce to one spatial dimension, the observed effects are entirely due to the temperature dependence of the material properties and not the result of any geometrical disturbance to the melt front.

Figure 15 shows the leading-order vertical velocity and mechanical dissipation across the melt layer. As predicted in [17], we see an increase in the velocity gradient near the sliding surface and as the temperature increases the velocity profile moves further away from the linear profile. We now observe that the leading-order dissipation is no longer constant in the melt layer, owing to the departure from the linear velocity profile. The dissipation term is computed as

$$
\Phi=\mu_{0}\left(\frac{\partial v_{0}}{\partial \zeta}\right)^{2}+\delta\left[2 \mu_{0} \frac{\partial v_{0}}{\partial \zeta} \frac{\partial V_{1}}{\partial \zeta}+\mu_{1}\left(\frac{\partial v_{0}}{\partial \zeta}\right)^{2}\right]+O\left(\delta^{2}\right)
$$

where we note that in the leading-order term both $\mu_{0}$ and $\partial v_{0} / \partial \zeta$ are now functions of $\zeta$ and $t$. This may be contrasted with the constant materials properties result in which the leading-order mechanical dissipation is a function of time only $\Phi=$ $\left(1 / X_{f 0}(t)\right)^{2}+O(\delta)$, see Section 3.1.

Figure 16 shows a comparison of the leading-order temperature and magnitude of the reaction source term for the constant material properties simulation (dashed blue) and temperature dependent material properties simulations (solid black). In panels $(\mathrm{a}-\mathrm{f})$ we only allow one of the material properties to very with temperature, allowing us to see how each property affects the shape of the leading-order temperature profile across the layer. Since the ignition times are changing, the plots are shown at approximately $25 \%, 50 \%, 75 \%$ and $100 \%$ of the time to thermal runaway, allowing the shapes of the temperature profiles to be compared. For the constant material properties simulation this corresponds to times $t=226,441,657$ and $872 \mathrm{~ns}$. For the temperature dependant plots times are: $t=206,403,599$ and $795 \mathrm{~ns}$ for temperature dependent specific heat only; $t=326,643,959$ and $1276 \mathrm{~ns}$ for temperature dependent viscosity only; $t=31,52,73$ and $94 \mathrm{~ns}$ for temperature dependent thermal conductivity only; and $t=117,223,330$ and 436 ns for temperature dependent specific heat, viscosity and thermal conductivity. Considering the large variation in time to runaway for each of the cases studied, it may be concluded that accurate modelling of the temperature dependence of the material properties is vital for making reasonable predictions about the time to runaway. However, in this paper we are concerned with how perturbations to the melt front, as well as the temperature dependence of the material properties, affect the spatial distribution of the temperature within the layer, and are less concerned with making predictions about runaway times.

Figure 16 (a) compares the temperature in the melt layer in the case of constant material properties with the solution found for temperature dependent specific heat. It is observed that the shape of the temperature profile remains nearly un- 
changed, due to the weak dependence of the specific heat on the temperature. However, we observe in Figures 16 (c/d) and (e/f) that allowing the viscosity and specific heat to vary with temperature alters the shape of the temperature profile. When $\mu=\mu(T)$ it is found that the hot spot is confined to a thinner region near the wall when compared with the constant material properties solution, due to the increased velocity gradient near the wall, see Figure 15. In contrast, when $\kappa=\kappa(T)$ the zone in which significant reaction occurs is comparatively much wider. This may seem counterintuitive, as one might expect decreased thermal conductivity lead to a more localised hot spot. However, in the early stages on melting, when the heating is dominated by viscous dissipation, it is found that the heat diffuses across the layer leading to more rapid melting in the constant material properties case. When we account for the temperature dependence of the specific heat the timescales are such that there isn't enough time for the temperature to diffuse across the entire layer before the reaction commences, leading to a wider region adjacent to the wall in which the temperature is elevated, ultimately leading to a wider reaction zone.

It is found that the introduction of temperature dependence in the specific heat, viscosity and thermal conductivity does not suppress the localisation mechanisms induced by perturbing the melt front, and it is still the case that the melt front may be unstable to small perturbations in the presence of chemical reaction. As an example case, Figure 17 shows results for HMX with an initial melt shape $S_{1}(y)$ with $\delta=0.2$. As found in the previous section, the imposed disturbance in the melt front causes a mechanical hot spot adjacent to the moving wall. This is most clearly seen in the middle pane which shows the magnitude of the reaction source term in the melt layer - we see that the reaction is starting to kick off all along the wall, owing the the increased mechanical heating near $X=0$, but there is clearly a localised hot spot opposite the site of the disturbance in the melt front. This highly localised heating ultimately leads to a substantial temperature increase and the time to ignition is significantly decreased when compared with the corresponding one-dimensional (or, equivalently, uniform width) case. As in the constant material properties case, it is found that the substantial temperature increase due to chemical reaction causes the initially decaying perturbation to the melt front to grow in time.

\section{Conclusion}

The numerical results presented here indicate that perturbations from a uniform width melt layer can cause localised heating due to mechanical dissipation. In particular, it has been demonstrated that accounting for the non-uniform melting behaviour through the introduction of spatial dependence in the melt front location can reduce the time to ignition compared with the corresponding one-dimensional model of melting.

In order to model the non-uniform melting, highly idealised melt front shapes were selected for analysis. The shapes chosen captured both of the possible important disturbances: those which locally increase the width of the melt layer, corresponding to areas which initially melted more quickly, and those which locally reduce the width of the melt layer, corresponding to areas which initially melted more slowly. One possible physical interpretation of this would be a scenario in which the melt temperature of the unmelted solid explosive material varies locally in space. This would result in a non-constant melt temperature, which would be accounted for in equation (13). This would affect the propagation of the melt front, 
causing some areas to melt rapidly, thus introducing some shape in the melt front. In our modelling it is assumed that the melt layer has already formed at time $t_{0}$, and it is reasoned that the initial melt front shape we impose could be generated by the mechanism described above. Local differences in other thermo-mechanical properties would operate in a similar fashion to give rise to non-uniform melting.

For certain disturbances it was shown that the time to ignition may be considerably reduced. For instance, the results in Figure 8 predicted an ignition time of $256 \mathrm{~ns}$ compared with an ignition time on the microsecond timescale for the corresponding one-dimensional simulation. The possibly reduced time to ignition predicted by the two-dimensional model increases the range of physical scenarios in which the shear melting hot spot mechanism is plausible.

For example, we may consider the physical scenario of an internal microcrack. In their analysis, Starobin and Dienes [17] find that for sliding speeds below $50 \mathrm{~m} \mathrm{~s}^{-1}$ the ignition time predicted by the one-dimensional model exceeds a millisecond. Over this time period the slip would be greater than $5 \mathrm{~cm}$, a distance much larger than the HMX grain size found in a plastic-bonded explosive (PBX), meaning the shear crack would cross the grain boundary [17]. The crossing of the boundary would likely change the crack propagation direction and the model with lateral melting would no longer be appropriate. However, due to local inhomogeneities in the material properties, the ignition time may be significantly reduced, even to the nanosecond timescale, meaning that the shear melting mechanism may lead to ignition in this model well before the slip length becomes an issue.

For the initial part of this study many material properties, such as viscosity, were assumed constant with respect to pressure and temperature. Later these assumptions were relaxed, and the the specific heat, viscosity and thermal conductivity were allowed to vary with temperature. As suggested by Starobin and Dienes [17], it was shown that the leading-order velocity profile was no longer linear, with most of the slip being supported in the region of the melt layer adjacent to the wall. This in turn introduced spatial dependence into the leading-order mechanical dissipation term. The pressure dependence of the material properties may also be studied. For example, Menikoff and Sewell [19] propose a modified form of the viscosity which increases with pressure, which may go some way to offset the drop in viscosity associated with the temperature increase across the layer.

Future work may include computation of numerical solutions for the fully-coupled governing equations, accounting for temperature (and possibly pressure) dependence of the material properties. This would allow for consideration of larger departures from the uniform melting case, but the results would be qualitatively similar to those found here. We would certainly expect the hot spot mechanisms introduced by the shape in the melt front to persist.

\section{Acknowledgement(s)}

This work was supported by an EPSRC industrial CASE partnership with AWE [grant number EP/L505729/1]. The authors gratefully acknowledge the comments of the reviewer(s), in particular the suggestion to extend the analysis to account for the temperature dependence of the thermal conductivity. 


\section{References}

[1] J. Field, N. Bourne, S. Palmer, S. Walley, J. Sharma, and B. Beard, Hot-spot ignition mechanisms for explosives and propellants [and discussion], Phil.Trans. of the R. Soc. Lond. A 339 (1992), pp. 269-283.

[2] F. Bowden, M. Mulcahy, R. Vines, and A. Yoffe, The detonation of liquid explosives by gentle impact - the effect of minute gas spaces, Proc. R. Soc. Lond. A 188 (1947), pp. 291-311.

[3] A. Ubbelohde, Part iii. (4) Mechanical and thermal processes of initiation, Phil.Trans. of the R. Soc. Lond. A 241 (1948), pp. 280-286.

[4] F. Bowden and O. Gurton, Initiation of solid explosives by impact and friction: The influence of grit, Proc. R. Soc. Lond. A 198 (1949), pp. 337-349.

[5] F. Bowden and A. Yoffe, Initiation and growth of explosions in liquids and solids, Cambridge University Press, 1952.

[6] Y. Bai and B. Dodd, Adiabatic shear localization: Occurrence, theories and applications (1992).

[7] J. DiLellio and W. Olmstead, Shear band formation due to a thermal flux inhomogeneity, SIAM Journal on applied mathematics 57 (1997), pp. 959-971.

[8] J. Field, G. Swallowe, and S. Heavens, Ignition mechanisms of explosives during mechanical deformation, Proc. R. Soc. Lond. A 382 (1982), pp. 231-244.

[9] V. Boyle, R. Frey, and O. Blake, Combined pressure shear ignition of explosives, in 9th Symposium (International) on Detonation, 1989, p. 3.

[10] H. Chen, V. Nesterenko, J. LaSalvia, and M. Meyers, Shear-induced exothermic chemical reactions, Le Journal de Physique IV 7 (1997), pp. 3-27.

[11] J. Dienes, On reactive shear bands, Physics Letters A 118 (1986), pp. 433-438.

[12] R. Frey, The initiation of explosive charges by rapid shear, Tech. Rep., DTIC Document, 1980.

[13] G. Afanas'ev and V. Bobolev, Initiation of solid explosives by impact, Israel Program for Scientific Translations, 1971.

[14] P. Howe, G. Gibbons Jr, and P. Webber, An experimental investigation of the role of shear in initiation of detonation by impact, Tech. Rep., DTIC Document, 1986.

[15] V.K. Mohan, V.J. Bhasu, and J. Field, Role of adiabatic shear bands in initiation of explosives by drop-weight impact, in 9th Symposium (International) on Detonation. Office of the Chief of Naval Research, Arlington, Virginia, 1989, pp. 1276-1283.

[16] J. Curtis, Explosive Ignition due to Adiabatic Shear, in 39th International Pyrotechnics Seminar, 2013.

[17] A. Starobin and J. Dienes, One-dimensional thermomechanical model for lateral melting and ignition of a thin sheared viscous layer, Combustion Theory and Modelling 10 (2006), pp. 885-905.

[18] R. Austin, N. Barton, J. Reaugh, and L. Fried, Direct numerical simulation of shear localization and decomposition reactions in shock-loaded hmx crystal, Journal of Applied Physics 117 (2015).

[19] R. Menikoff and T. Sewell, Constituent properties of HMX needed for mesoscale simulations, Combustion theory and modelling 6 (2002), pp. 103-125.

[20] D. Bedrov, G.D. Smith, and T.D. Sewell, Thermal conductivity of liquid octahydro-1,3,5,7tetranitro-1,3,5,7-tetrazocine (HMX) from molecular dynamics simulations, Chemical Physics Letters 324 (2000), pp. 64-68.

[21] H. Ockendon and J. R., Viscous flow, Vol. 13, Cambridge University Press, 1995.

[22] M. Abramowitz, I. Stegun, et al., Handbook of mathematical functions, Applied Mathematics Series 55 (1966), p. 62.

[23] H. Carslaw and J. Jaeger, Conduction of heat in solids, Oxford: Clarendon Press (1959). 
Table 1. Material properties for HMX taken from $[16,17,19]$.

\begin{tabular}{cc}
\hline Explosive Property & HMX \\
\hline Density $\rho$ & $1860 \mathrm{~kg} \mathrm{~m}^{-3}$ \\
Reference Specific Heat $c^{*}$ & $1034 \mathrm{~J} \mathrm{~kg}^{-1} \mathrm{~K}^{-1}$ \\
Reference Thermal Conductivity $\kappa^{*}$ & $0.3884 \mathrm{~W} \mathrm{~m}^{-1} \mathrm{~K}^{-1}$ \\
Reference Viscosity $\mu^{*}$ & $5.5 \times 10^{-3} \mathrm{~kg} \mathrm{~m}^{-1} \mathrm{~s}^{-1}$ \\
Melting Temperature $T_{\mathrm{m}}$ & $520.6 \mathrm{~K}$ \\
Latent Heat $L$ & $2.08 \times 10^{5} \mathrm{~J} \mathrm{~kg}^{-1}$ \\
Activation Energy $E$ & $2.2 \times 10^{5} \mathrm{~J} \mathrm{~mol}^{-1}$ \\
Heat of Reaction $\Omega$ & $5.02 \times 10^{6} \mathrm{~J} \mathrm{~kg}^{-1}$ \\
Molar Gas Constant $R$ & $8.314 \mathrm{~J} \mathrm{~mol}^{-1} \mathrm{~K}^{-1}$ \\
Pre-Exponential Constant $A$ & $5.011872336 \times 10^{19} \mathrm{~s}^{-1}$ \\
\hline
\end{tabular}

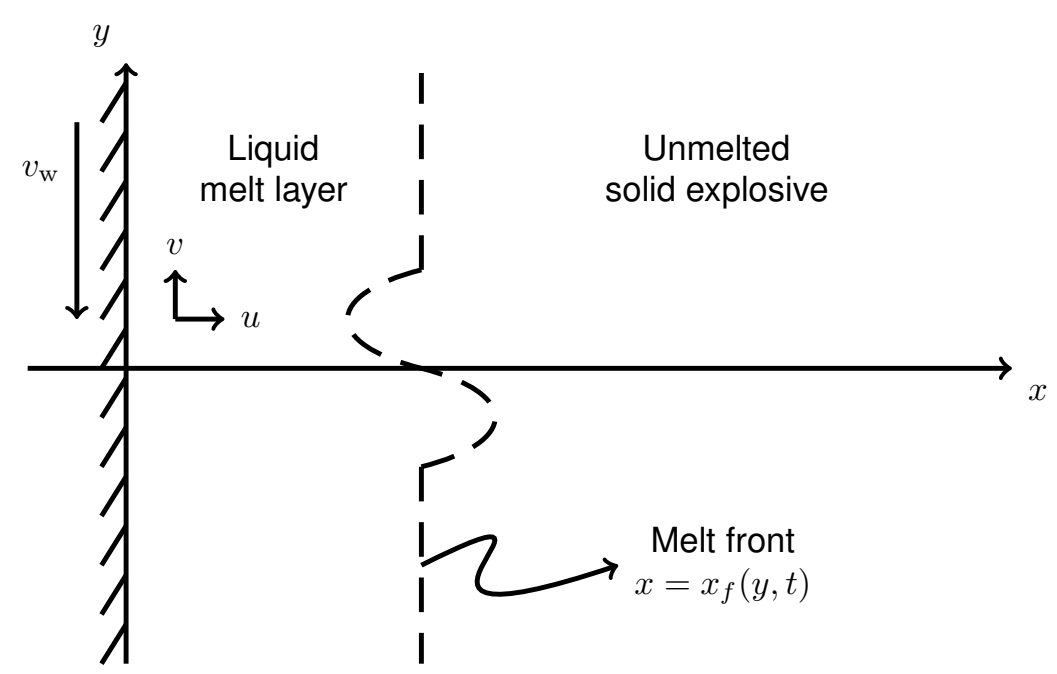

Figure 1. A solid block of explosive material occupies the region $x>0$. At $t=0$ the rigid wall located at $x=0$ is impulsively moved downward, so that at $t=t_{0}$ a thin viscous liquid melt layer has already formed.
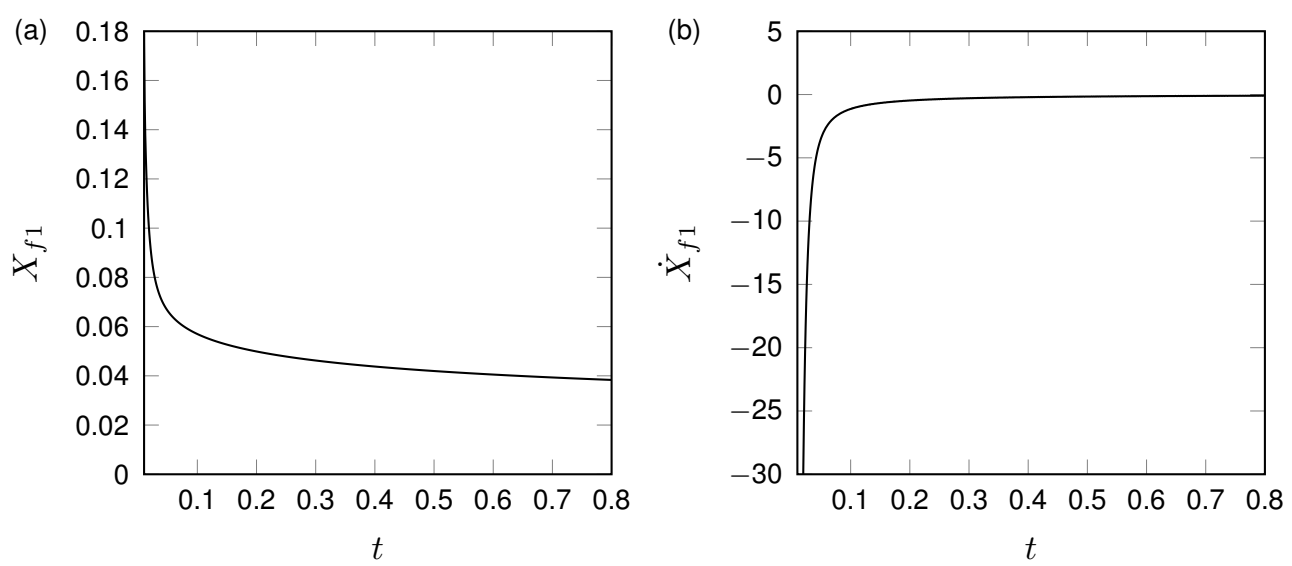

Figure 2. (a) Melt front correction $X_{f 1}$; and (b) melt front correction propagation speed $\dot{X}_{f 1}$ for an inert melt layer, both as a function of non-dimensional time $t$. In the absence of any chemical reaction, the melt front is shown to be linearly stable to small perturbations in shape. 
(a)

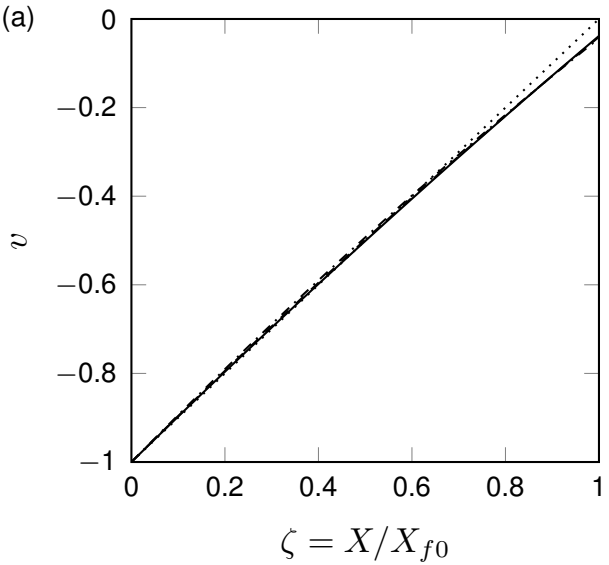

(b)

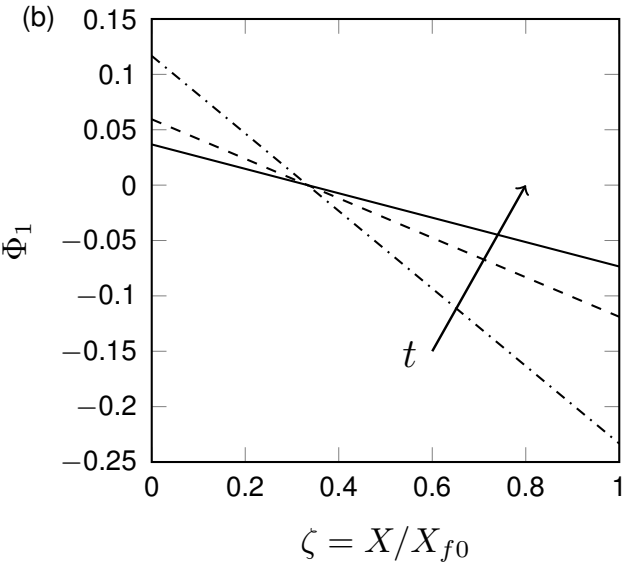

Figure 3. (a) The vertical velocity $v$ correct up to $O(\delta)$; and (b) the $(\xi, t)$ dependence of the first-order correction to the mechanical dissipation, i.e. $\Phi_{1}=4\left(X_{f 1} / X_{f 0}^{3}\right)(1-3 \zeta) S(y)$ with fixed $S(y)=1$. Both are plotted as a function of the leading -order front-fixed variable $\zeta$ at a series of increasing times. The dotted line $(\cdots)$ in $(\mathrm{a})$ shows the leading-order linear velocity profile.
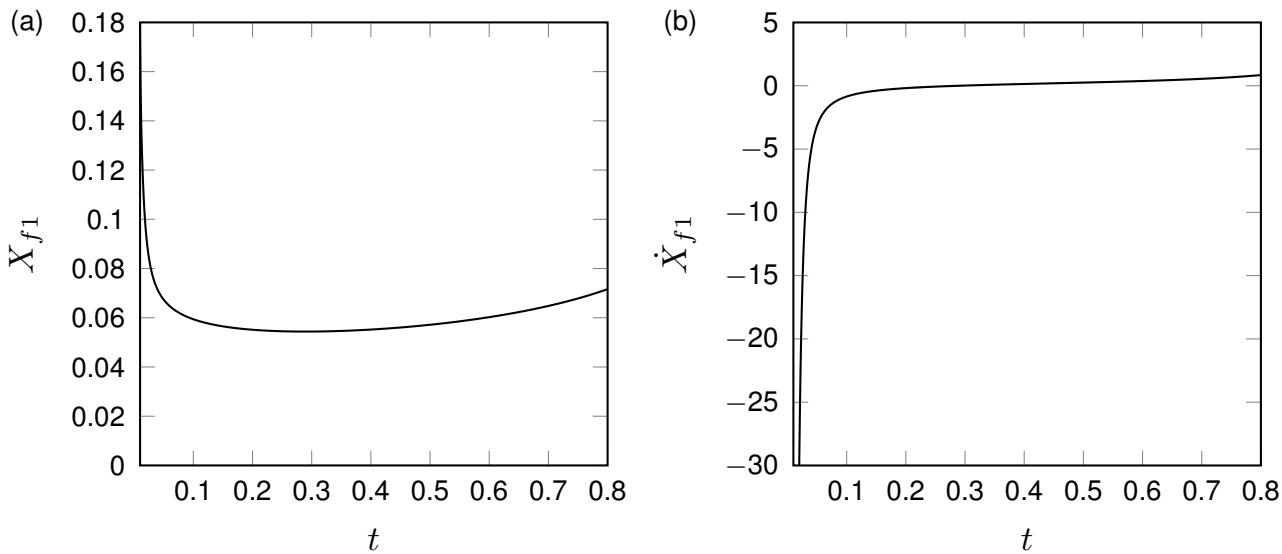

Figure 4. (a) Melt front correction $X_{f 1}$; and (b) melt front correction propagation speed $\dot{X}_{f 1}$ for a reactive melt layer, both as a function of non-dimensional time $t$. It is found the the inclusion of a chemical reaction in the melt layer has a destabilising effect; the melt front is no longer linearly stable to small perturbations in shape. 


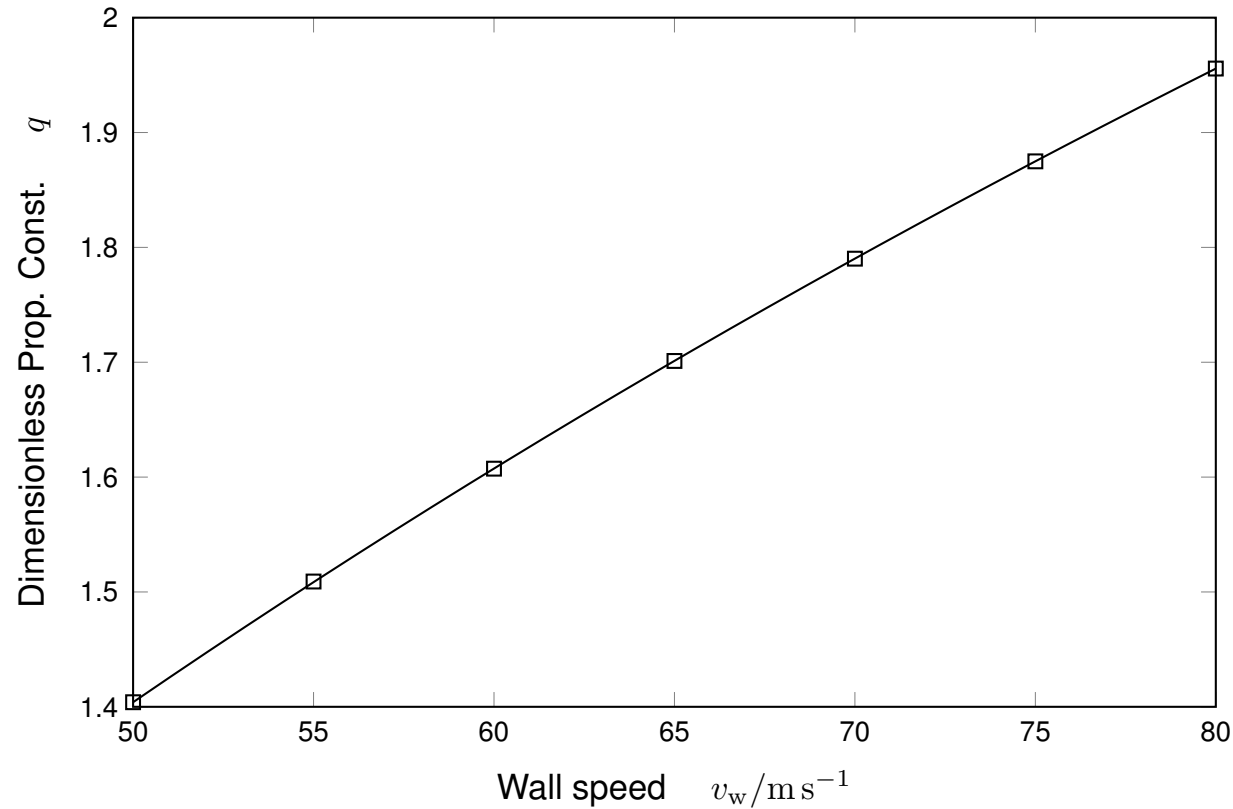

Figure 5. Dimensionless propagation constant $q$ as a function of dimensional wall speed $v_{\mathrm{w}}$.
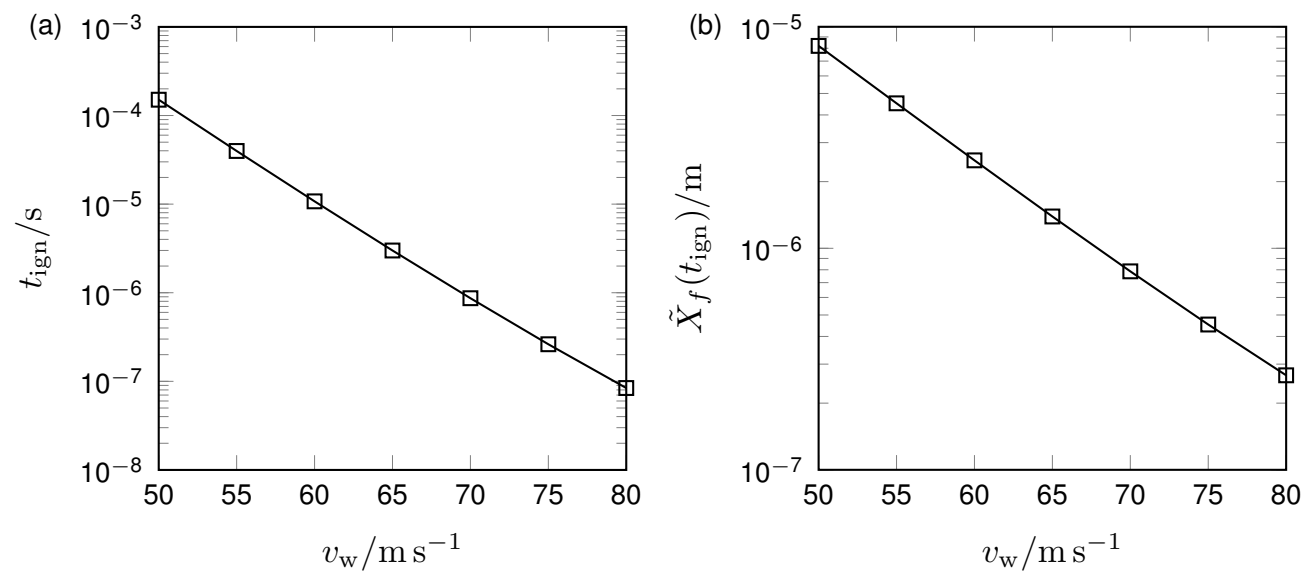

Figure 6. (a) Dimensional ignition time $t_{\text {ign }}$; and (b) dimensional melt width at ignition $\tilde{X}_{f}$, both as a function of dimensional wall speed $v_{\mathrm{w}}$ for a melt layer of uniform width in HMX. 
(a)

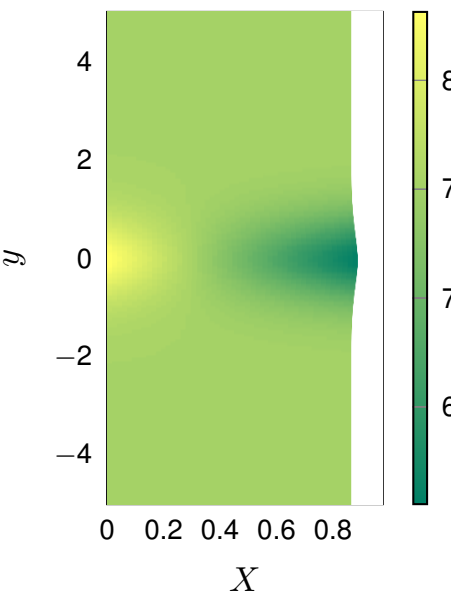

(b)

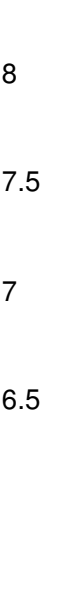

$\hat{\Omega} \dot{\alpha}$

(c)

$T / \mathrm{K}$

Figure 7. (a) Non-dimensional instantaneous energy increase due to mechanical dissipation; (b) nondimensional instantaneous energy increase due to reaction; and (c) dimensional temperature (K) of a sample of HMX at $90 \%$ of the time to runaway since $t_{0}$. An initial melt front shape $S_{1}(0.8, y)$ and wall speed $v_{\mathrm{w}}=70 \mathrm{~m} \mathrm{~s}^{-1}$ were used. [Original in colour.]

(a)

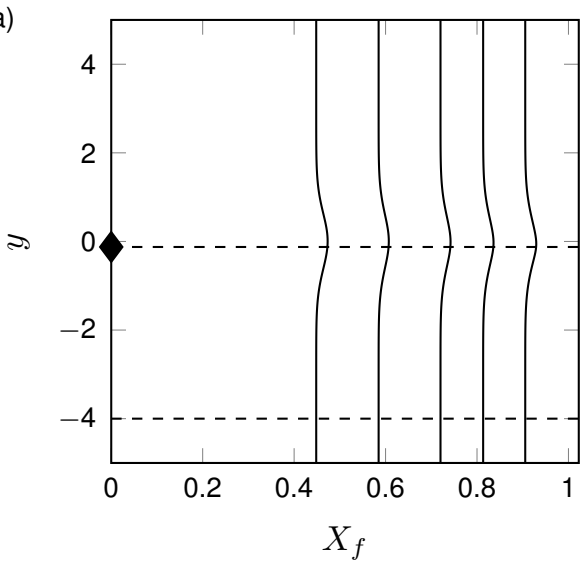

(b)

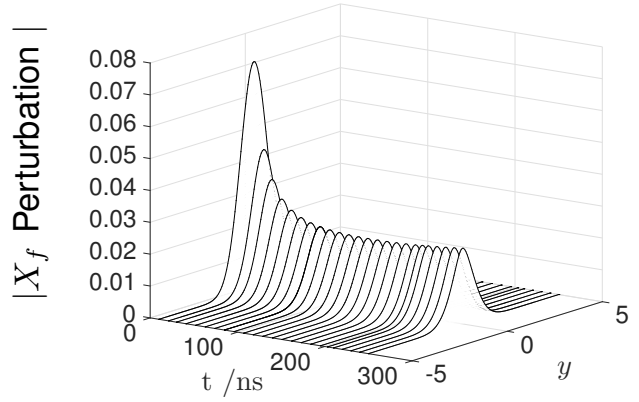

(c) 1,0

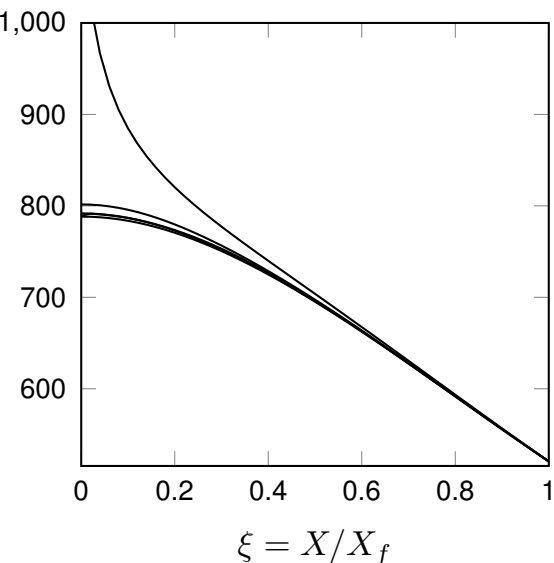

(d) 1,0

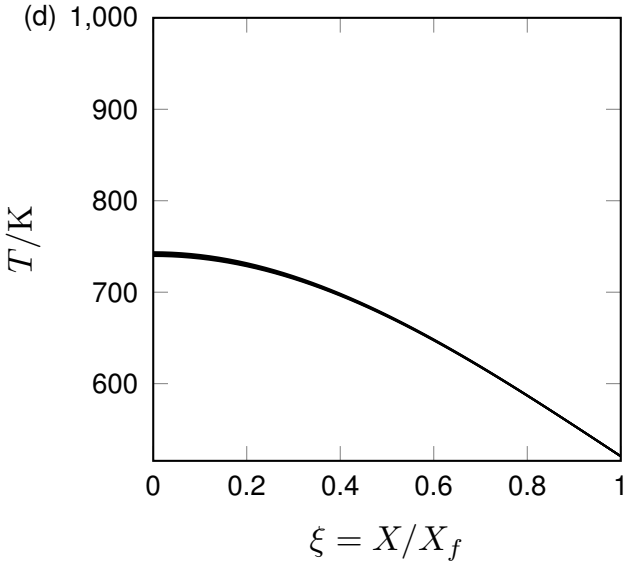

Figure 8. (a) Melt front location at times $t=59,108,158,207,256 \mathrm{~ns}$ for a sample of HMX with initial melt front shape $S_{1}(0.8, y)$; and (b) the magnitude of the melt front perturbation at a series of increasing times. Also shown are temperature profiles across the melt width at the times depicted in (a), plotted at vertical coordinates: (c) $y=y_{\mathrm{R}}$, where $y_{\mathrm{R}}$ is the vertical coordinate of the peak reaction site, and (d) $y=-4$. The filled diamond symbol shows the location of the peak reaction site at ignition. The dashed lines in (a) show the locations of the temperature profiles taken in (c) and (d). A wall speed $v_{\mathrm{w}}=70 \mathrm{~m} \mathrm{~s}^{-1}$ was used. 
(a)

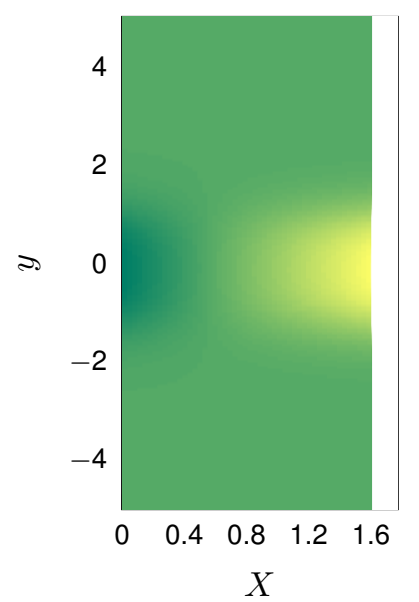

(b)

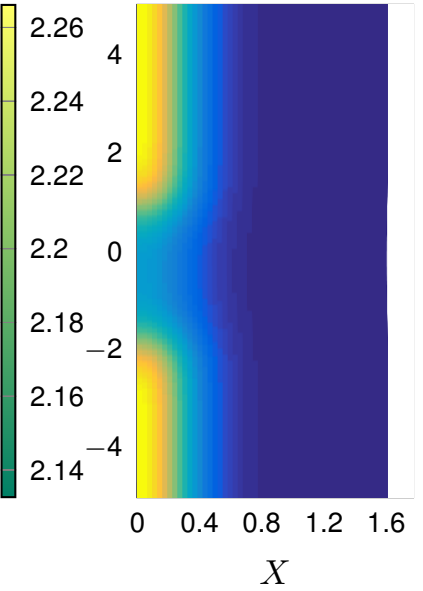

(c)

$T / \mathrm{K}$

Figure 9. (a) Non-dimensional instantaneous energy increase due to mechanical dissipation; (b) nondimensional instantaneous energy increase due to reaction; and (c) dimensional temperature (K) of a sample of HMX at $90 \%$ of the time to runaway since $t_{0}$. An initial melt front shape $S_{1}(-0.8, y)$ and wall speed $v_{\mathrm{w}}=70 \mathrm{~ms}^{-1}$ were used. [Original in colour.]

(a)

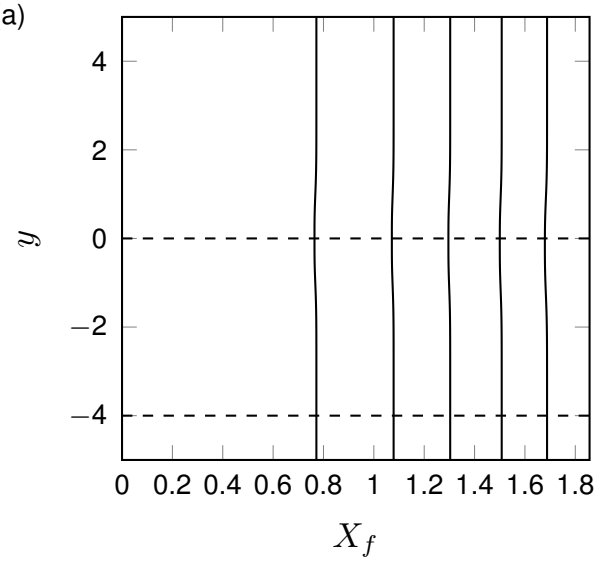

(b)

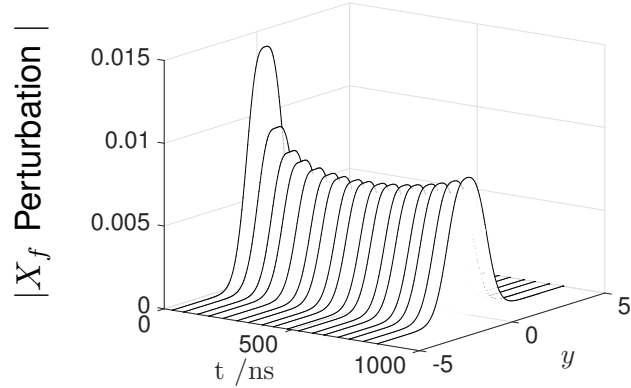

(c) 1 ,

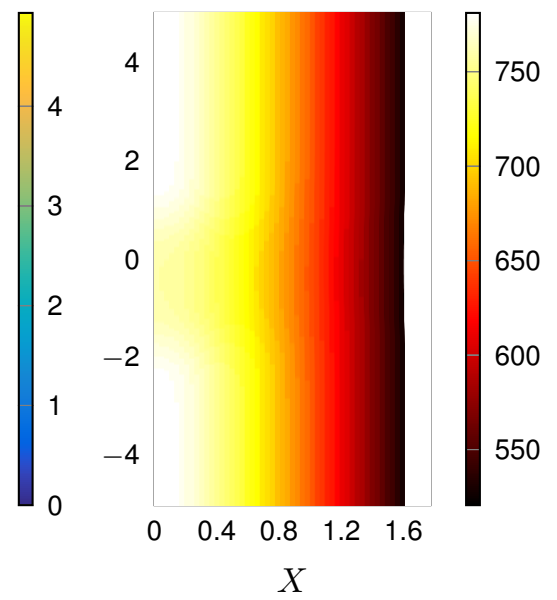

50

00

50

00 
(a)

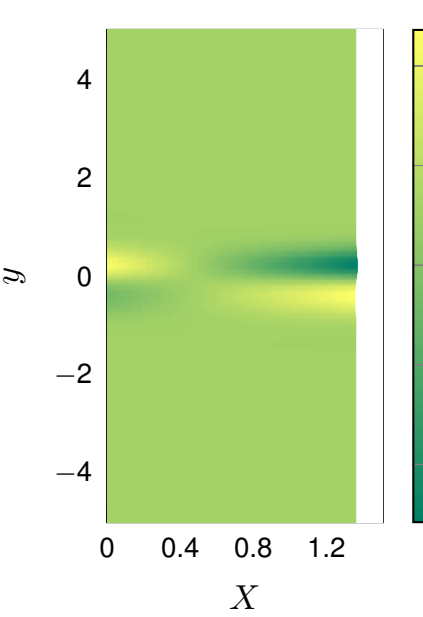

(b)

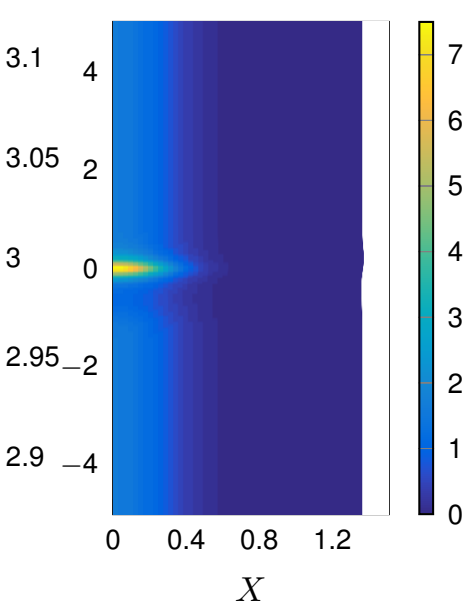

(c)

$T / \mathrm{K}$

Figure 11. (a) Non-dimensional instantaneous energy increase due to mechanical dissipation; (b) nondimensional instantaneous energy increase due to reaction; and (c) dimensional temperature (K) of a sample of HMX at $90 \%$ of the time to runaway since $t_{0}$. An initial melt front shape $S_{2}(0.5, y)$ and wall speed $v_{\mathrm{w}}=70 \mathrm{~m} \mathrm{~s}^{-1}$ were used. [Original in colour.]

(a)

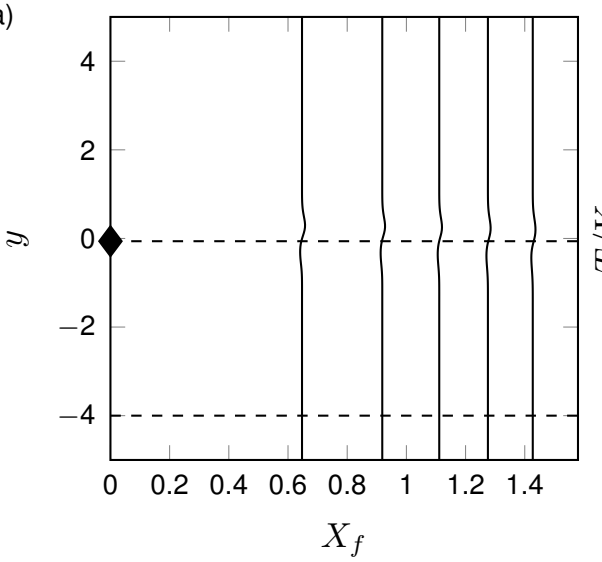

(b)

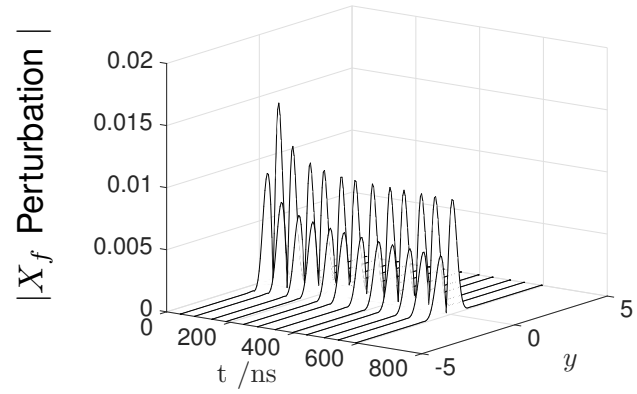

(c) 1,00
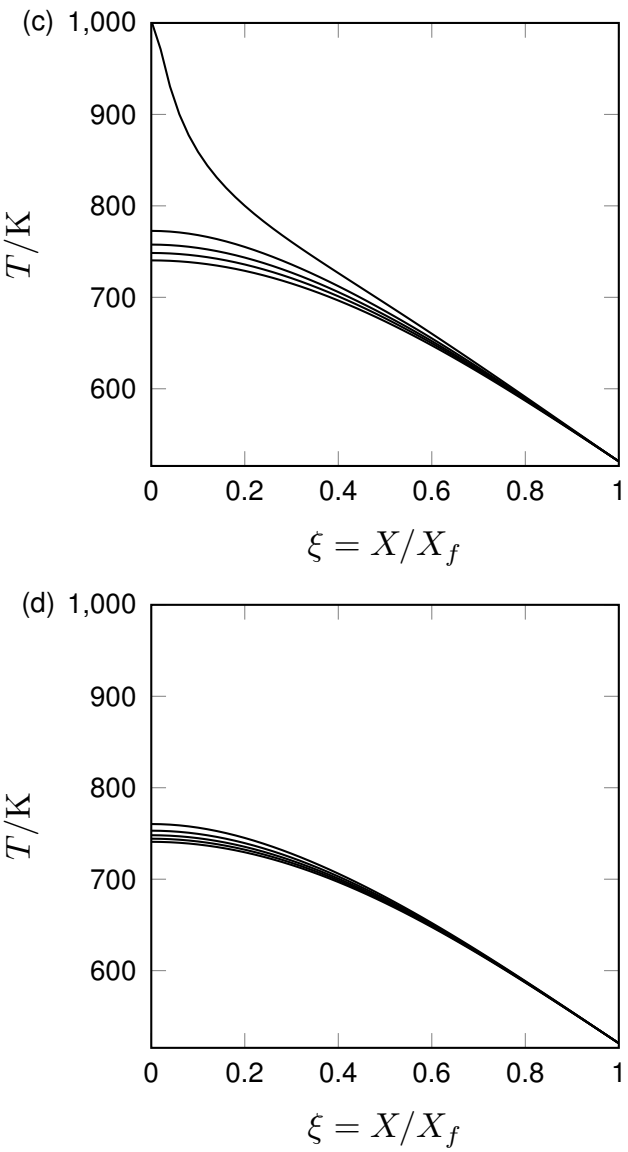

Figure 12. Melt front location at times $t=134,258,383,507,631 \mathrm{~ns}$ for a sample of HMX with initial melt front shape $S_{2}(0.5, y)$; and (b) the magnitude of the melt front perturbation at a series of increasing times. Also shown are temperature profiles across the melt at the times depicted in (a), plotted at vertical coordinates: (c) $y=y_{\mathrm{R}}$, where $y_{\mathrm{R}}$ is the vertical coordinate of the peak reaction site, and (d) $y=-4$. The filled diamond symbol shows the location of the peak reaction site at ignition. The dashed lines in (a) show the locations of the temperature profiles taken in (c) and (d). A wall speed $v_{\mathrm{w}}=70 \mathrm{~m} \mathrm{~s}^{-1}$ was used. 


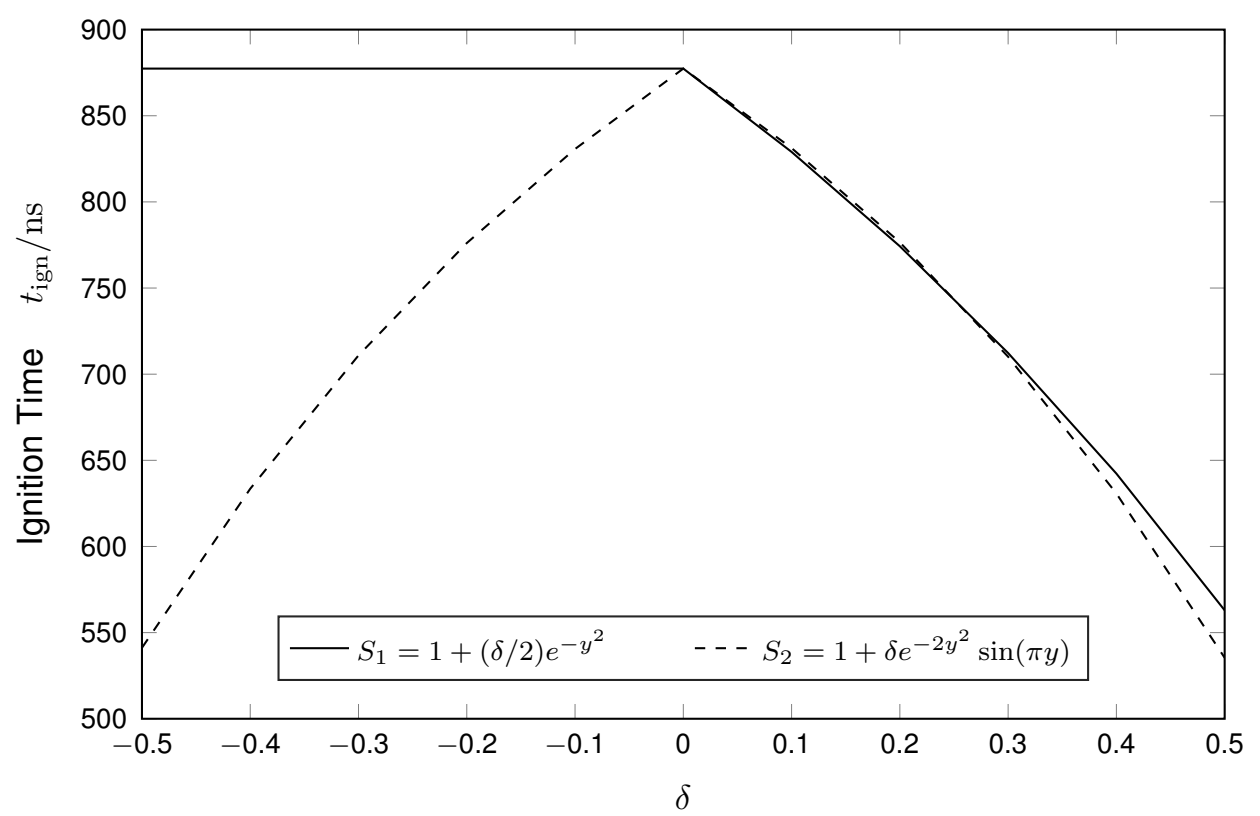

Figure 13. Time to ignition $t_{\text {ign }}$ as a function of $\delta$ for initial melt front shapes $S_{1}$ and $S_{2}$.
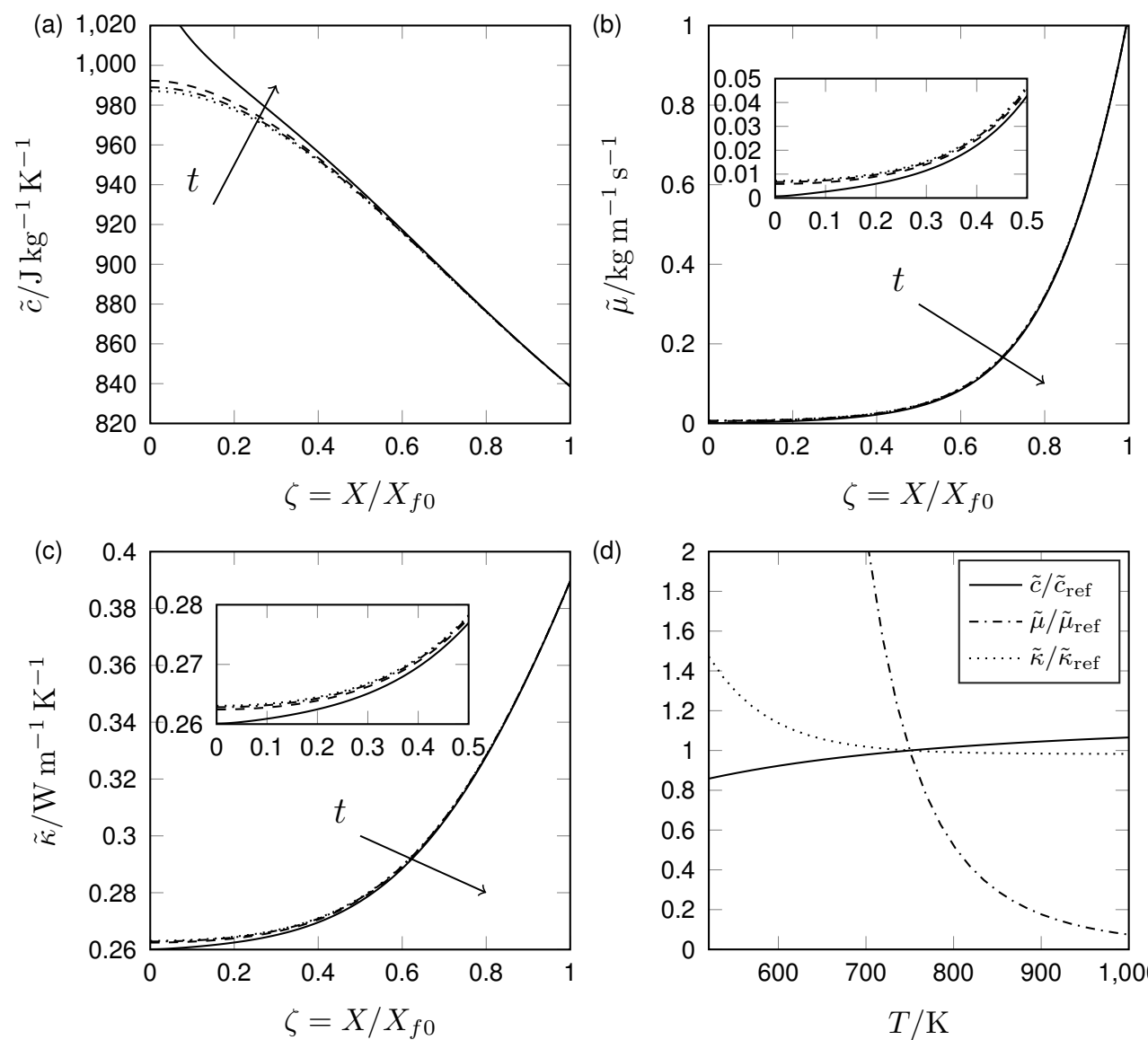

(d)

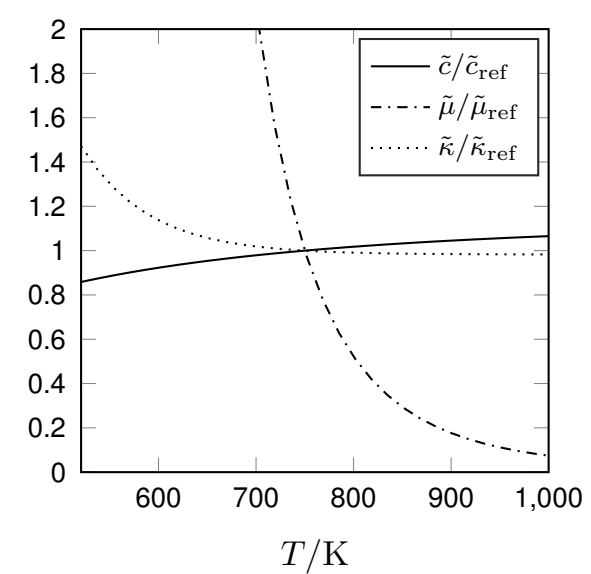

Figure 14. (a) The leading-order dimensional specific heat $\tilde{c}=c^{*} c_{0}$; (b) the leading-order dimensional viscosity $\tilde{\mu}=\mu^{*} \mu_{0}$; and (c) the leading-order dimensional thermal conductivity $\tilde{\kappa}=\kappa^{*} \kappa_{0}$ across the melt layer at times $t=117,223,330$ and $436 \mathrm{~ns}$. Panel (d) shows the material properties as a function of temperature, scaled by their values at a reference temperature of $750 \mathrm{~K}$. 
(a)

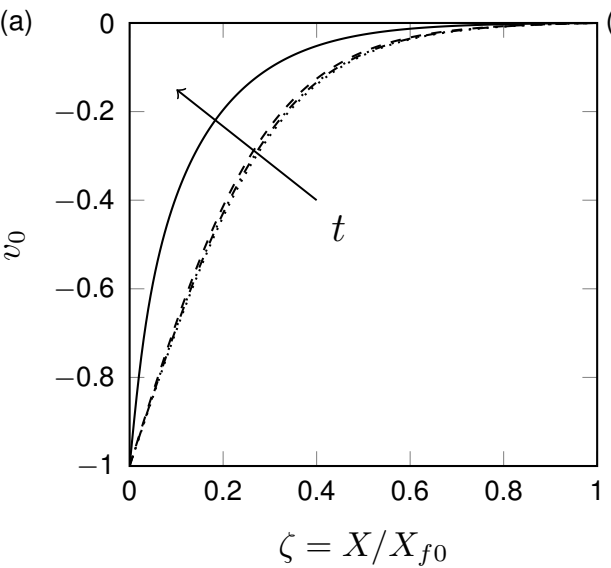

(b)

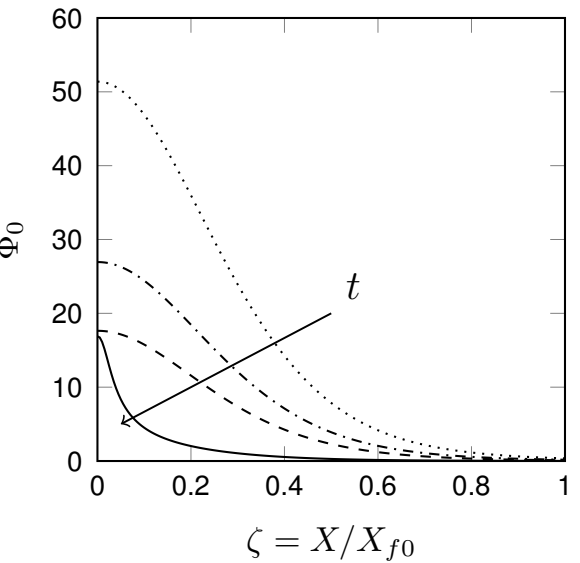

Figure 15. (a) The leading-order vertical velocity profile $v_{0}$ across the melt layer at times $t=117,223,330$ and $436 \mathrm{~ns}$; and (b) The non-dimensional leading-order energy increase due to viscous dissipation across the melt layer shown at the same times. 

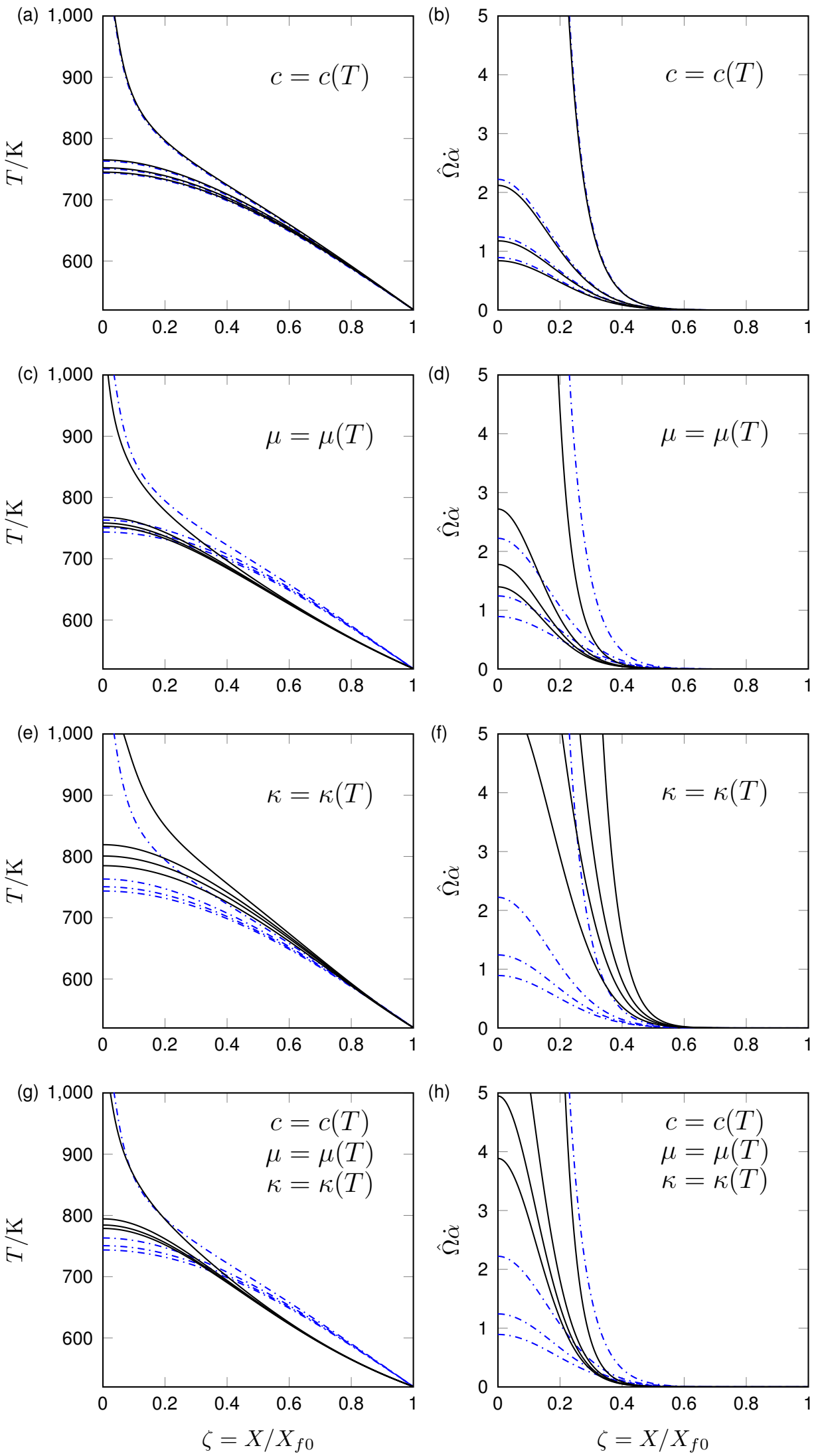

Figure 16. (a,c,e,g) The dimensional leading-order temperature across the melt layer; and (b,d,f,h) the non-dimensional leading-order energy increase due to chemical reaction across the melt layer. The dashed blue line shows results with constant material properties in each plot. The solid black line shows results for: $(\mathrm{a} / \mathrm{b})$ temperature dependent specific heat; (c/d) temperature dependent viscosity; (e/f) temperature dependent thermal conductivity; and $(\mathrm{g} / \mathrm{h})$ temperature dependent specific heat, viscosity and thermal conductivity. The plots show snapshots at approximately $25 \%, 50 \%, 75 \%$ and $100 \%$ of the time to thermal runaway. For the constant material properties simulation this corresponds to times $t=226,441,657$ and 872 ns. For the temperature dependant plots the times shown are: (a/b) $t=206,403,599$ and 795 ns; (c/d) $t=326,643,959$ and $1276 \mathrm{~ns} ;(\mathrm{e} / \mathrm{f}) t=31,52,73$ and $94 \mathrm{~ns} ;$ and $(\mathrm{g} / \mathrm{h}) t=117,223,330$ and $436 \mathrm{~ns}$. [Original in colour.] 
(a)

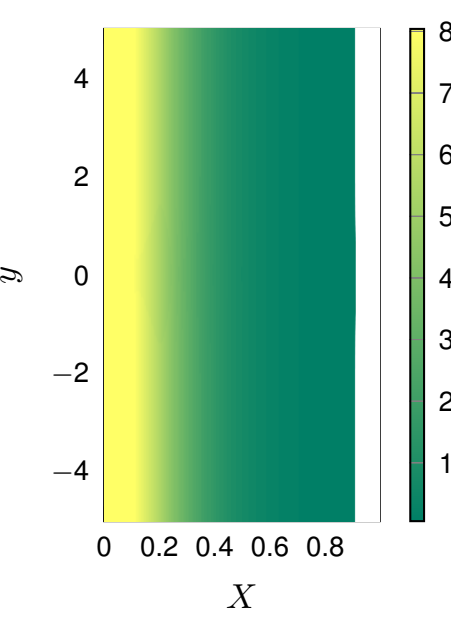

(b)

$\Omega \alpha$

(c)

$T / \mathrm{K}$

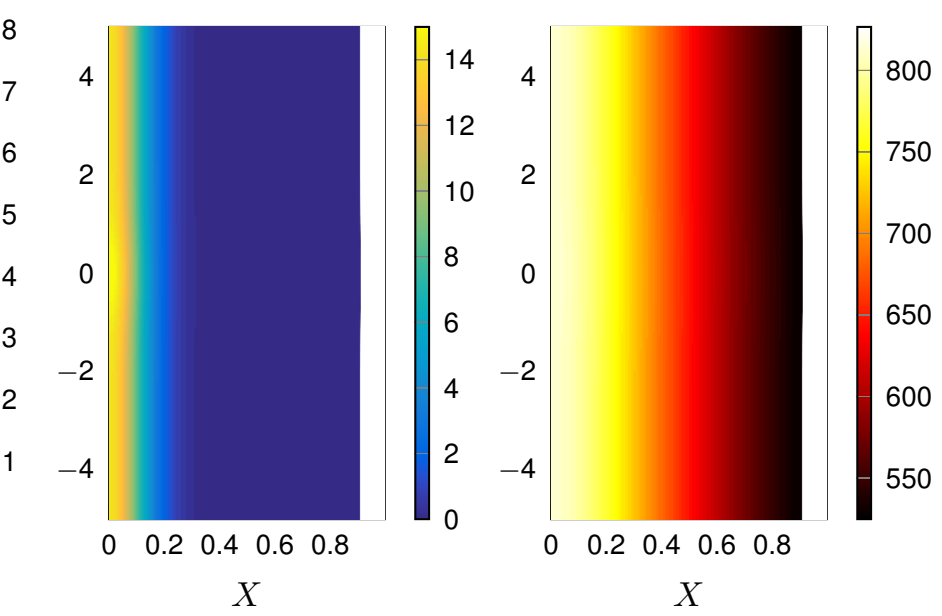

Figure 17. (a) Non-dimensional instantaneous energy increase due to mechanical dissipation; (b) nondimensional instantaneous energy increase due to reaction; and (c) dimensional temperature (K) of a sample of HMX at $90 \%$ of the time to runaway since $t_{0}$, as predicted by the analysis which includes temperature dependent specific heat and viscosity. An initial melt front shape $S_{1}(0.2, y)$ and wall speed $v_{\mathrm{w}}=70 \mathrm{~m} \mathrm{~s}^{-1}$ were used. [Original in colour.] 


\section{Appendix A. Small-time behaviour}

Shortly after the onset of melting we expect a balance between the rate of change of temperature and the horizontal diffusion term in the liquid region, essentially reducing the problem to one which is locally one-dimensional. Here we show that we recover the model presented in [17] by considering the two-dimensional model at early times. For $t \ll 1$ and $x_{f} \ll 1$ we anticipate the following scalings

$$
t \sim \delta \ll 1, \quad x \sim \sqrt{t} \sim \delta^{1 / 2} \ll 1, \quad u \sim \delta^{1 / 2} \ll 1, \quad y \sim 1, \quad v \sim 1
$$

At early times we make the assumption that the velocity field is linear $v=x / x_{f}-1$, giving local viscous heating $\left(1 / x_{f}\right)^{2}$. Note that the large Prandtl number $\operatorname{Pr} \approx 110$ indicates that thermal diffusion time is much greater than the momentum diffusion time, and validates our assumption that the velocity field is near the steady state [17]. Further, in the early stages of melting the temperature in the melt layer is not expected to be near the activation temperature for the reaction, so the Arrhenius source term is exponentially small.

Under these early time assumptions, and the assumption of constant material properties, the model equations have a solution for a parabolic advancement law for the phase boundary $x_{f}=q \sqrt{t}$ which is equivalent to the one-dimensional solution presented in [17]. The solutions in the liquid and solid region are given here in terms of the front-fixed variable $\xi=x / x_{f}=x /(q \sqrt{t})$.

In each phase, the temperature may be written as a sum of of two functions

$$
T(\xi, t)=f(\xi, t)+h_{1}(\xi), \quad T_{\mathrm{sol}}(\xi, t)=f(\xi, t)+h_{2}(\xi)
$$

The functions $h_{i}(\xi)$ satisfy the time-independent form of the governing equations, along with the appropriate boundary conditions, and are given by

$$
\begin{aligned}
& h_{1}(\xi)=T_{m}+\operatorname{Ec} \operatorname{Pr} \frac{\sqrt{\pi}}{2 u}\left(\operatorname{erf}(\sqrt{u}) \int_{0}^{\sqrt{u}} e^{q^{2}} \mathrm{~d} q-\int_{0}^{\sqrt{u}} \operatorname{erf}(q) e^{q^{2}} \mathrm{~d} q\right) \\
& -\operatorname{Ec} \operatorname{Pr} \int_{0}^{\xi} e^{-u \xi_{2}^{2}} \int_{0}^{\xi_{2}} e^{u \xi_{1}^{2}} \mathrm{~d} \xi_{1} \mathrm{~d} \xi_{2}, \quad 0<\xi<1,
\end{aligned}
$$

and

$$
h_{2}(\xi)=\bar{T}-\left(\bar{T}-T_{m}\right) \frac{\operatorname{erfc}(\sqrt{u} \xi)}{\operatorname{erfc}(\sqrt{u})}, \quad \xi>1,
$$

respectively. The Stefan condition provides the equation for the propagation parameter $u$

$$
\operatorname{Pe} x_{f} \dot{x}_{f}=2 u=\operatorname{Ste}\left(\operatorname{Ec} \operatorname{Pr} \frac{e^{-u}}{\sqrt{u}} \int_{0}^{\sqrt{u}} e^{q^{2}} \mathrm{~d} q-\frac{2\left(T_{\mathrm{m}}-\bar{T}\right)}{\sqrt{\pi}} \frac{e^{-u} \sqrt{u}}{\operatorname{erfc}(\sqrt{u})}\right) .
$$

The function $f(\xi, t)$ must satisfy have vanishing derivative at the origin, decay at infinity and be continuous at the melt front. It can be shown that $f$ has the 
following form:

$$
f(\zeta, t) \propto\left(\frac{t_{0}}{t}\right)^{\beta^{2} / q^{2}}{ }_{1} F_{1}\left(\frac{\beta^{2}}{q^{2}}, \frac{1}{2},-\frac{q^{2}}{4} \xi^{2}\right),
$$

where $t_{0}$ is the initial time, ${ }_{1} F_{1}$ is a confluent hypergeometric function [22], and $\beta$ is a constant which is fixed by ensuring the $f(\xi, t)$ vanishes at $\xi=1$ (thus ensuring $T=T_{\mathrm{m}}=T_{\text {sol }}$ at $\xi=1$ ).

A number of other self similar solutions with a parabolic advancement law for the phase boundary are available. For discussion see Carslaw and Jaeger [23], for example.

\section{Appendix B. Discrete approximations}

The discrete approximations to the differential operators used in the numerical scheme read

$$
\begin{aligned}
\left\{\frac{\partial T}{\partial t}\right\}_{i, j}^{k+1 / 2} & =\frac{T_{i, j}^{k+1}-T_{i, j}^{k}}{\Delta t}, \\
\left\{\frac{\partial T}{\partial \xi}\right\}_{i, j}^{k+1 / 2}= & \frac{1}{2}\left(\frac{T_{i+1, j}^{k+1}-T_{i-1, j}^{k+1}}{2 \Delta \xi}+\frac{T_{i+1, j}^{k}-T_{i-1, j}^{k}}{2 \Delta \xi}\right), \\
\left\{\frac{\partial^{2} T}{\partial \xi^{2}}\right\}_{i, j}^{k+1 / 2}= & \frac{1}{2}\left(\frac{T_{i+1, j}^{k+1}-2 T_{i, j}^{k+1}+T_{i-1, j}^{k+1}}{(\Delta \xi)^{2}}+\frac{T_{i+1, j}^{k}-2 T_{i, j}^{k}+T_{i-1, j}^{k}}{(\Delta \xi)^{2}}\right), \\
\left\{\frac{\partial T}{\partial y}\right\}_{i, j}^{k+1 / 2}= & \frac{1}{2}\left(\frac{T_{i, j}^{k+1}-T_{i, j-1}^{k+1}}{2 \Delta y}+\frac{T_{i, j+1}^{k}-T_{i, j-1}^{k}}{2 \Delta y}\right), \\
\left\{\frac{\partial v}{\partial \xi}\right\}_{i, j}^{k+1 / 2}= & \frac{1}{2}\left(\frac{{ }^{*} v_{i+1, j}^{k+1}-{ }^{*} v_{i-1, j}^{k+1}}{2 \Delta \xi}+\frac{v_{i+1, j}^{k}-v_{i-1, j}^{k}}{2 \Delta \xi}\right), \\
\left\{\exp \left(-\frac{\hat{E}}{T}\right)\right\}_{i, j}^{k+1 / 2}= & \exp \left(-\frac{2 \hat{E}}{\left({ }^{*} T_{i, j}^{k+1}+T_{i, j}^{k}\right)}\right)
\end{aligned}
$$

where the pre-superscript asterix indicates that the previous iterate should be used in order to linearise the problem. 